\title{
PHASE-FIELD APPROXIMATION FOR A CLASS OF COHESIVE FRACTURE ENERGIES WITH AN ACTIVATION THRESHOLD
}

\author{
ANTONIN CHAMBOLLE AND VITO CRISMALE \\ CMAP, École Polytechnique, 91128 Palaiseau Cedex, France
}

\begin{abstract}
We study the $\Gamma$-limit of Ambrosio-Tortorelli-type functionals $D_{\varepsilon}(u, v)$, whose dependence on the symmetrised gradient $e(u)$ is different in $\mathbb{A} u$ and in $e(u)-\mathbb{A} u$, for a $\mathbb{C}$-elliptic symmetric operator $\mathbb{A}$, in terms of the prefactor depending on the phase-field variable $v$. The limit energy depends both on the opening and on the surface of the crack, and is intermediate between the Griffith brittle fracture energy and the one considered by Focardi and Iurlano in [39]. In particular we prove that $G(S) B D$ functions with bounded $\mathbb{A}$-variation are $(S) B D$.
\end{abstract}

\section{Contents}

1. Introduction

2. Notation and a preliminary result

3. $\Gamma$-liminf inequality 11

4. $\Gamma$-lim sup inequality 16

References

\section{INTRODUCTION}

The energy functionals in Fracture Mechanics are usually expressed in terms of the displacement $u: \Omega \subset \mathbb{R}^{n} \rightarrow \mathbb{R}^{n}$ as the sum of a volume part, accounting for the mechanical properties of the uncracked material in the bulk region, and of a surface part, concentrated on a $(n-1)$-dimensional discontinuity set of $u$ (the crack set) and representing the energy dissipated in the crack process.

The presence of the crack set entails difficulties in the effective computation of minimisers, for instance by numerical simulations. A possible, and by now classical, way out is to approximate the energy in the sense of $\Gamma$-convergence, through simpler functionals. These depend on the two variables $u: \Omega \rightarrow \mathbb{R}^{n}$, which is now a Sobolev function and represents the regularised displacement, and the phase-field $v: \Omega \rightarrow[0,1]$, whose sublevels $\{v<s\}$, for $s \in(0,1)$, may be used to approximate the limit discontinuity set. Such approximations are often called of Ambrosio-Tortorelli type, from the breakthrough paper [9] they realised to approximate the Mumford-Shah functional [52] in image reconstruction.

In the context of Fracture Mechanics, Ambrosio-Tortorelli approximations are largely employed since the Francfort-Marigo's work [42] on the variational approach to fracture

E-mail address: antonin.chambolle@cmap.polytechnique.fr, vito.crismale@polytechnique.edu. 2010 Mathematics Subject Classification. 49J45, 26A45, 49Q20, 74R99, 35Q74.

Key words and phrases. free discontinuity problems, $\Gamma$-convergence, special functions of bounded deformation, cohesive fracture. 
and the first numerical experiments [16] (see e.g. [15, 5, 1] and references therein). The first case that has been considered is the Griffith energy [48]

$$
\int_{\Omega} f_{p}(e(u)) \mathrm{d} x+\mathcal{H}^{n-1}\left(J_{u} \cup\left(\partial_{D} \Omega \cap\left\{\operatorname{tr}_{\partial \Omega} u \neq \operatorname{tr}_{\partial \Omega} u_{0}\right\}\right)\right)
$$

where $u_{0} \in W^{1, p}\left(\mathbb{R}^{n} ; \mathbb{R}^{n}\right)$ enforces a Dirichlet boundary condition (by penalising $\operatorname{tr}_{\partial \Omega} u$, the trace on $\partial \Omega$ of $u$, where different from that of $u_{0}$ on the Dirichlet boundary $\partial_{D} \Omega$ ), $e(u):=\frac{\nabla u+\nabla u^{T}}{2} \in \mathbb{M}_{\text {sym }}^{n \times n}$ is the linearised strain (in the bulk) in small strain assumptions, $J_{u}$ is the jump set of $u$ (see Section 2), $\mathcal{H}^{n-1}$ is the $(n-1)$-dimensional Hausdorff measure, and $f_{p}: \mathbb{M}_{\text {sym }}^{n \times n} \rightarrow[0,+\infty)$ is convex with

$$
f_{p}(0)=0, \quad C_{f_{p}}\left(|\xi|^{p}-1\right)<f_{p}(\xi)<C_{f_{p}}^{\prime}\left(|\xi|^{p}+1\right), \quad p>1,
$$

for the Frobenius norm $|\cdot|$ on $\mathbb{M}_{s y m}^{n \times n}$. As explained e.g. in [29, Section 1] and [49, Sections 10 and 11], the reference form for $f_{p}$ is for every $\mu>0$

$$
f_{p, \mu}(\xi):=\frac{1}{p}\left((\Sigma \xi: \xi+\mu)^{\frac{p}{2}}-\mu^{\frac{p}{2}}\right),
$$

where $\Sigma$, such that $\Sigma\left(\xi-\xi^{T}\right)=0$ and $\Sigma \xi \cdot \xi \geq c_{0}\left|\xi+\xi^{T}\right|^{2}$ for all $\xi \in \mathbb{M}_{\text {sym }}^{n \times n}$, is the fourth-order Hooke's tensor: this is a slight generalisation of the original Griffith energy, where the bulk energy is the linear elastic energy, that is $p=2, \mu=0$ and

$$
\Sigma \xi \cdot \xi=\frac{1}{4} \lambda_{1}\left|\xi+\xi^{T}\right|^{2}+\frac{1}{2} \lambda_{2}(\operatorname{Tr} \xi)^{2},
$$

with $\lambda_{1}, \lambda_{2}$ the Lamé coefficients. The $f_{p, \mu}$ are quadratic for small $\xi$ and with $p$-growth for large $\xi$, and for $p \neq 2$ this may account for plastic deformation at large strain.

The Griffith energy $(\mathrm{G})$ is approximated by the functionals

$$
\int_{\Omega}\left(\left(v+\eta_{\varepsilon}\right) f_{p}(e(u))+\frac{(1-v)^{2}}{4 \varepsilon}+\varepsilon^{q-1}|\nabla v|^{q}\right) \mathrm{d} x, \quad \lim _{\varepsilon \rightarrow 0} \frac{\eta_{\varepsilon}}{\varepsilon^{p-1}}=0,
$$

for $u \in W_{u_{0}}^{1, p}\left(\Omega ; \mathbb{R}^{n}\right):=W^{1, p}\left(\Omega ; \mathbb{R}^{n}\right) \cap\left\{u: \operatorname{tr} \partial \Omega\left(u-u_{0}\right)=0\right.$ on $\left.\partial_{D} \Omega\right\}, v \in W_{1}^{1, q}(\Omega ;[0,1]):=$ $W^{1, q}(\Omega ;[0,1]) \cap\left\{v: \operatorname{tr}_{\partial \Omega} v=1\right.$ on $\left.\partial_{D} \Omega\right\}$, and $+\infty$ otherwise: such approximation has been proven without any a priori assumption on $u$, for any $p>1$, and in any dimension in 25], together with compactness for minimisers (see [26]), assuming that

$$
O_{\delta, x_{0}}\left(\partial_{D} \Omega\right) \subset \Omega \quad \text { for } \delta \in(0, \bar{\delta}),
$$

for some $\bar{\delta}>0$ and $x_{0} \in \mathbb{R}^{n}$, where $O_{\delta, x_{0}}(x):=x_{0}+(1-\delta)\left(x-x_{0}\right)$. This generalises [21, 22, 51, assuming a priori $u \in L^{2}$ and $p=2$, [28], requiring $u \in L^{p}, p>1$, and [43], obtained in dimension 2 (see also e.g. [45, 18, 53] for the antiplane shear case and different approximations).

In [39] Focardi and Iurlano studied the limit of the functionals

$$
\int_{\Omega}\left((v+\varepsilon) f_{2,0}(e(u))+\frac{\psi(v)}{\varepsilon}+\varepsilon^{q-1}|\nabla v|^{q}\right) \mathrm{d} x,
$$

for $u \in H^{1}\left(\Omega ; \mathbb{R}^{n}\right), v \in W^{1, q}(\Omega ;[0,1])$, and $+\infty$ otherwise (with $\psi \in C([0,1])$ decreasing, $\psi(1)=0)$ and proved that they $\Gamma$-converge to

$$
\int_{\Omega} f_{2,0}(e(u)) \mathrm{d} x+c_{1} \mathcal{H}^{n-1}\left(J_{u}\right)+c_{2} \int_{J_{u}}\left|[u] \odot \nu_{u}\right| \mathrm{d} \mathcal{H}^{n-1},
$$

for suitable $c_{1}, c_{2}>0$. The energy space for (C) is $S B D^{2}$, a subspace of the Special Bounded Deformation functions $S B D$ (see Section 2). For $v \in S B D$, the distributional gradient $\mathrm{E} v:=\frac{\mathrm{D} v+\mathrm{D}^{T} v}{2}$ is a bounded Radon measure, $J_{v}$ is the set of points $x$ at which 
$v$ has two different approximate limits $v^{+}(x), v^{-}(x)$ with respect to a suitable direction $\nu_{v}(x)$, and $[v](x):=v^{+}(x)-v^{-}(x)$ is the jump. We denote by $\odot$ the symmetrised tensor product, and notice that $[u] \odot \nu_{u}$ is the part of the total strain Eu concentrated on $J_{u}$, see (2.2).

The energy (C) depends also on the jump amplitude, reflecting mechanical interaction between the fracture lips. This is typical of cohesive fracture energies, in contrast to the brittle energy (G). On the other hand, (C) has not the form of the classical cohesive fracture energies in Barenblatt's model [13], which in particular do not depend on $\mathcal{H}^{n-1}\left(J_{u}\right)$. The presence of the measure of the crack surface corresponds to an activation energy which is necessary to nucleate the crack: this is considered also in [2, where it is called "depinning energy", in [4], that studies a model for quasistatic evolution, and in the approximation result [12]. A few others have succeeded in approximating particular instances of pure cohesive energies, as in [50, 27, 35], see also [3] (in these works the bulk energy is a function of the full gradient $\nabla u$ ).

In this work we approximate fracture energies that, as (C), include the measure of $J_{u}$, but whose cohesive term now depends only on a part of the strain, for instance on its deviatoric part (for $n \geq 3$ ). Moreover, we consider general $p$-growth $(p>1)$ in $e(u)$ of the bulk energy, no integrability assumptions on $u$, and study the Dirichlet boundary problem. To present the general case we consider a constant-coefficient, linear, first order differential operator

$$
\mathbb{A} u=\sum_{j=1}^{n} A_{j} \partial_{j} u, \quad u: \mathbb{R}^{n} \rightarrow \mathbb{R}^{n},
$$

for $A_{j} \in \mathcal{L}\left(\mathbb{R}^{n}, \mathbb{M}^{n \times n}\right)$ linear mappings. We assume that $\left(A_{j}\right)_{i}=\left(A_{i}\right)_{j}$ and that $\mathbb{A} u: \mathbb{R}^{n} \rightarrow$ $\mathbb{M}_{\text {sym }}^{n \times n}$, so that there is an endomorphism $A$ of $\mathbb{M}_{\text {sym }}^{n \times n}$ for which

$$
\mathbb{A} u=A(e(u)) \text {. }
$$

A Fourier symbol mapping $\mathbb{A}[z]: \mathbb{R}^{n} \rightarrow \mathbb{M}_{\text {sym }}^{n \times n}$ is introduced for every $z=\left(z_{1}, \ldots, z_{n}\right) \in \mathbb{R}^{n}$, defined by

$$
\mathbb{A}[z] v:=v \otimes_{\mathbb{A}} z:=\sum_{j=1}^{n} z_{j} A_{j} v=A([v] \odot z)
$$

for $v \in \mathbb{R}^{n}$; the operator $\mathbb{A}$ is $\mathbb{R}$-elliptic if $\mathbb{A}[z]$ is injective for all $z \in \mathbb{R}^{n} \backslash\{0\}$, and $\mathbb{C}$-elliptic if (take the estension of $\mathbb{A}[z] v$ on $\mathbb{C}^{n}$ ) $\mathbb{A}[z]: \mathbb{C}^{n} \rightarrow \mathbb{C}^{n \times n}$ is injective for all $z \in \mathbb{C}^{n} \backslash\{0\}$. These operators have been recently considered in e.g. [17, 46, 47, 37, 10, 57]. The deviator operator $\mathrm{E}_{D} u:=\mathrm{E} u-\frac{1}{n}(\operatorname{div} u) \operatorname{Id}_{n}$ is $\mathbb{C}$-elliptic for $n \geq 3$, but not for $n=2$ (see Remark 2.5).

From a mechanical point of view, the reference problem is to minimise the energy $F$ under a Dirichlet boundary condition on a part of the boundary $\partial_{D} \Omega \neq \emptyset$ with possibly the presence of volume forces, and surface forces on the remaining part of the boundary $\partial_{N} \Omega$, with

$$
\partial \Omega=\partial_{D} \Omega \cup \partial_{N} \Omega \cup N, \quad \partial_{D} \Omega \cap \partial_{N} \Omega=\emptyset, \quad \mathcal{H}^{n-1}(N)=0, \quad \partial\left(\partial_{D} \Omega\right)=\partial\left(\partial_{N} \Omega\right),
$$

for $\partial_{D} \Omega$ and $\partial_{N} \Omega$ relatively open. Here we assume all forces null, 1.2 , and that

$$
\lim _{s \rightarrow \pm \infty} \frac{f_{p}(s \xi)}{|s|^{p}}=\tilde{f}_{p}(\xi) \quad \text { uniformly as } s \rightarrow \pm \infty, \xi \in \mathbb{M}_{s y m}^{n \times n}
$$

We have that $\tilde{f}_{p}$ is positively $p$-homogeneous, and $\left(\tilde{f}_{p}\right)^{\frac{1}{p}}$ is a norm on $\mathbb{M}_{\text {sym }}^{n \times n}$ (cf. e.g. [40, Remark 2.7]). Then we prove the following, main result of this work.

Theorem 1.1. Let $\Omega \subset \mathbb{R}^{n}$ be open bounded Lipschitz satisfying 1.2 , $1.6, u_{0} \in$ $W^{1, p}\left(\mathbb{R}^{n} ; \mathbb{R}^{n}\right), p, q>1, \gamma>0, \varepsilon>0, \eta_{\varepsilon}>0$ such that $\lim _{\varepsilon \rightarrow 0} \frac{\eta_{\varepsilon}}{\varepsilon^{p-1}}=0, f_{p}$ and $\tilde{f}_{p}$ 
satisfying $\left(\overline{\mathrm{HP} 1 f_{p}}\right),\left(\overline{\mathrm{HP} 2 f_{p}}\right), \psi \in C([0,1])$ decreasing with $\psi(1)=0$, and $\mathbb{A}$ be $\mathbb{C}$-elliptic. Then the functionals $\overline{D_{\varepsilon}(u, v)}$ defined on $u, v$ measurable by

$$
D_{\varepsilon}(u, v):=\int_{\Omega}\left[\left(v+\varepsilon^{p-1}\right) f_{p}(\mathbb{A} u)+\left(v+\eta_{\varepsilon}\right) f_{p}(e(u)-\mathbb{A} u)+\frac{\psi(v)}{\varepsilon}+\gamma \varepsilon^{q-1}|\nabla v|^{q}\right] \mathrm{d} x
$$

if $u \in W_{u_{0}}^{1, p}\left(\Omega ; \mathbb{R}^{n}\right), v \in W_{1}^{1, q}(\Omega ;[0,1])$ and by $+\infty$ otherwise, $\Gamma$-converge, as $\varepsilon \rightarrow 0$, to

$$
\begin{aligned}
D(u, v):=\int_{\Omega} & {\left[f_{p}(A(e(u)))+f_{p}(e(u)-A(e(u)))\right] \mathrm{d} x+\int_{J_{u}}\left[a+b\left(\tilde{f}_{p}\right)^{\frac{1}{p}}\left([u] \otimes_{\mathbb{A}} \nu_{u}\right)\right] \mathrm{d} \mathcal{H}^{n-1} } \\
& +\int_{\partial_{D} \Omega \cap\left\{\operatorname{tr}_{\partial \Omega}\left(u-u_{0}\right) \neq 0\right\}}\left[a+b\left(\tilde{f}_{p}\right)^{\frac{1}{p}}\left(\operatorname{tr}_{\partial \Omega}\left(u-u_{0}\right) \otimes_{\mathbb{A}} \nu_{\partial \Omega}\right)\right] \mathrm{d} \mathcal{H}^{n-1},
\end{aligned}
$$

if

$$
u \in S B D^{p}(\Omega), \quad v=1 \text { a.e. in } \Omega,
$$

and by $+\infty$ otherwise for $u, v$ measurable, with respect to the topology of convergence in $\mathcal{L}^{n}$ measure for $u$ and $v$. Above $A$ is the operator introduced in (1.4), and $\left(\frac{1}{p^{\prime}}+\frac{1}{p}=\frac{1}{q^{\prime}}+\frac{1}{q}=1\right.$ )

$$
a:=2\left(q^{\prime}\right)^{1 / q^{\prime}}(\gamma q)^{1 / q} \int_{0}^{1} \psi^{1 / q^{\prime}}, \quad b:=p^{1 / p}\left(p^{\prime}\right)^{1 / p^{\prime}} \psi(0)^{1 / p} .
$$

Moreover, for every $M>0$ and $\varepsilon<1$, the sublevel $\left\{(u, v): D_{\varepsilon}(u, v) \leq M\right\}$ is contained in

$$
\left\{(u, v): \int_{\Omega}|\mathbb{A} u| \mathrm{d} x \leq C_{M}, \operatorname{tr}_{\partial \Omega} u=\operatorname{tr}_{\partial \Omega} u_{0} \text { on } \partial_{D} \Omega, \int_{\Omega} \psi(v) \mathrm{d} x \leq M \varepsilon\right\} .
$$

Then a sequence of quasi-minimisers for $D_{\varepsilon}$ converge, up to a subsequence, to a minimiser of $D$, with respect to the product of the strong $L^{r}\left(\Omega ; \mathbb{R}^{n}\right)$ topology for $u$, for any $r \in\left[1, \frac{n}{n-1}\right)$, times the topology of convergence in $\mathcal{L}^{n}$-measure for $v$, provided $\mathcal{H}^{n-1}\left(\partial_{D} \Omega \cap \partial \Omega_{j}\right)>0$, for each $\Omega_{j}$ connected component of $\Omega$.

The functionals $f_{p, \mu}$ in (1.1) satisfy $\left(\operatorname{HP} 2 f_{p}\right)$, and $f_{2, \mu}(\mathbb{A} u)+f_{2, \mu}(e(u)-\mathbb{A} u)=f_{2, \mu}(e(u))$ if $\mathbb{A}=\mathrm{E}_{D}$, so, in this case, we recover the linear elastic energy in the bulk.

Our approximating functionals are in some sense intermediate between those in $\left(\mathrm{G}_{\varepsilon}\right)$ and $\left(\overrightarrow{\mathrm{C}_{\varepsilon}}\right)$, since the part corresponding to $e(u)-\mathbb{A} u$ is multiplied by $v+\eta_{\varepsilon}$ as in $\left(\mathrm{G}_{\varepsilon}\right)$, while $f_{p}(\mathbb{A} u)$ is multiplied by $v+\varepsilon^{p-1}$ as in $\left(\mathrm{C}_{\varepsilon}\right)$ for $p=2$. This results in an interaction between $\left(v+\varepsilon^{p-1}\right) f_{p}(\mathbb{A} u)$ and $\frac{\psi(v)}{\varepsilon}$ that gives the term in $[u]$ in the limit. As usual, the surface of $J_{u}$ is approximated by the Ambrosio-Tortorelli part $\frac{\psi(v)}{\varepsilon}+\gamma \varepsilon^{q-1}|\nabla v|^{q}$.

Since the integrals of $\left(v+\eta_{\varepsilon}\right) f_{p}(e(u)-\mathbb{A} u)$ and $\frac{\psi(v)}{\varepsilon}$ are not energetically of the same order, we have an a priori control only on $\mathbb{A} u$ as a Radon measure, differently from [39], where this control is on the whole Eu. For this reason we initially work in the space $G S B D$ of generalised $S B D$ functions, introduced by Dal Maso in 34 to study brittle fracture (in [39] the control on Eu allowed to work directly in $S B D)$. A crucial point is to establish the expression of $\mathbb{A} u$ on the set $J_{u}$, in particular to show that

$$
\mathbb{A} u\left\llcorner J_{u}=[u] \otimes_{\mathbb{A}} \nu_{u} \mathcal{H}^{n-1}\left\llcorner J_{u}\right.\right.
$$

$\left(J_{u},[u], \nu_{u}\right.$ are well defined in $G S B D$, see Section 2): we prove this equality employing the tools developed in [17, 46, 47] to show the existence of a trace for functions with bounded $\mathbb{A}$-variation if and only if $\mathbb{A}$ is $\mathbb{C}$-elliptic. This is enough to conclude that $G S B D$ functions with bounded $\mathbb{A}$-variation are in fact in $S B D$, because we deduce that $[u]$ is integrable on $J_{u}$ (this is also true for $G B D, B D$ in place of $G S B D, S B D$, see Theorem 2.9). Technical problems arise to prove the same in dimension 2 for $\mathbb{A}=\mathrm{E}_{D}$ (see Remark 2.10).

We remark that in [24] the approximating functionals weight differently $\mathrm{E}_{D} u+\operatorname{div}^{+} u$ (multiplied by $(v+\epsilon)$ ) and $\operatorname{div}^{-} u$ (without any prefactor), so that $\operatorname{div}^{-} u$ is equibounded in $L^{2}$ and in the limit $[u] \cdot \nu \geq 0$, a linearised non-interpenetration condition. Here we could 
also separate the behaviours of $\operatorname{div}^{+} u$ and $\operatorname{div}^{-} u$ (Remarks 3.2 and 4.6 ), but the meaning of our approach is in some sense opposite to [24], since we do not pay, in the limit part in $[u]$, for the terms multiplied by $v+\eta_{\varepsilon}$, while in [24] the concentration of terms without prefactor pay infinite energy. One might also consider a non-interpenetration condition in our model, for instance by studying the $\Gamma$-limit of (for Id the identity $n \times n$ matrix)

$$
\int_{\Omega}\left[\left(v+\varepsilon^{p-1}\right) f_{p}\left(\mathrm{E}_{D} u\right)+\left(v+\eta_{\varepsilon}\right) f_{p}\left(\operatorname{div}^{+} u \mathrm{Id}\right)+f_{p}\left(\operatorname{div}^{-} u \mathrm{Id}\right)+\frac{\psi(v)}{\varepsilon}+\gamma \varepsilon^{q-1}|\nabla v|^{q}\right] \mathrm{d} x,
$$

but the $\Gamma$-limsup inequality presents hard difficulties. In this respect, we point out that for the $\Gamma$-lim sup inequality in Theorem 1.1] we employ the approximation result [31] for $S B D^{p}$ functions in $B D$-norm, which allows us to prove the result without any regularity assumption on the displacement, as the uniform $L^{\infty}$ bound in [39]. We have also to refine the argument of [31], in order to deal with Dirichlet boundary conditions.

\section{Notation AND A PRELIMinary RESUlt}

We denote by $\mathcal{L}^{n}$ and $\mathcal{H}^{k}$ the $n$-dimensional Lebesgue measure and the $k$-dimensional Hausdorff measure. For any locally compact subset $B$ of $\mathbb{R}^{n}$, the space of bounded $\mathbb{R}^{m}$ valued Radon measures on $B$ is indicated as $\mathcal{M}_{b}\left(B ; \mathbb{R}^{m}\right)$. For $m=1$ we write $\mathcal{M}_{b}(B)$ for $\mathcal{M}_{b}(B ; \mathbb{R})$ and $\mathcal{M}_{b}^{+}(B)$ for the subspace of positive measures of $\mathcal{M}_{b}(B)$. For every $\mu \in \mathcal{M}_{b}\left(B ; \mathbb{R}^{m}\right),|\mu|(B)$ stands for its total variation. We use the notation: $B_{\varrho}(x)$ [and $Q_{\varrho}(x)$ ] for the open ball [cube] with center $x$ and radius [sidelength] $\varrho ; x \cdot y,|x|$ for the scalar product and the norm in $\mathbb{R}^{n} ; 1^{*}$ for $n /(n-1), n$ being the space dimension; $\mathrm{d}(x, A)$ for the distance of $x$ from $A ; A \Subset K$ when $A$ compactly contained in $K$.

We recall the definition of approximate limit with respect to the convergence in measure and approximate jump set for measurable functions.

Definition 2.1. Let $A \subset \mathbb{R}^{n}, v: A \rightarrow \mathbb{R}^{m}$ an $\mathcal{L}^{n}$-measurable function, $x \in \mathbb{R}^{n}$ such that

$$
\limsup _{\varrho \rightarrow 0^{+}} \frac{\mathcal{L}^{n}\left(A \cap B_{\varrho}(x)\right)}{\varrho^{n}}>0 .
$$

A vector $a \in \mathbb{R}^{n}$ is the approximate limit of $v$ as $y$ tends to $x$ if for every $\varepsilon>0$

$$
\lim _{\varrho \rightarrow 0^{+}} \frac{\mathcal{L}^{n}\left(A \cap B_{\varrho}(x) \cap\{|v-a|>\varepsilon\}\right)}{\varrho^{n}}=0,
$$

and then we write

$$
\underset{y \rightarrow x}{\operatorname{ap} \lim } v(y)=a .
$$

Definition 2.2. Let $U \subset \mathbb{R}^{n}$ open, and $v: U \rightarrow \mathbb{R}^{m}$ be $\mathcal{L}^{n}$-measurable. The approximate jump set $J_{v}$ is the set of points $x \in U$ for which there exist $a, b \in \mathbb{R}^{m}$, with $a \neq b$, and $\nu \in \mathbb{S}^{n-1}$ such that

$$
\underset{(y-x) \cdot \nu>0, y \rightarrow x}{\operatorname{ap} \lim } v(y)=a \text { and } \quad \underset{(y-x) \cdot \nu<0, y \rightarrow x}{\operatorname{ap} \lim } v(y)=b .
$$

The triplet $(a, b, \nu)$ is uniquely determined up to a permutation of $(a, b)$ and a change of sign of $\nu$, and is denoted by $\left(v^{+}(x), v^{-}(x), \nu_{v}(x)\right)$. The jump of $v$ is the function defined by $[v](x):=v^{+}(x)-v^{-}(x)$ for every $x \in J_{v}$. 
$B V$ and $B D$ functions. For $U \subset \mathbb{R}^{n}$ open, a function $v \in L^{1}(U)$ is a function of bounded variation on $U$, denoted by $v \in B V(U)$, if $\mathrm{D}_{i} v \in \mathcal{M}_{b}(U)$ for $i=1, \ldots, n$, where $\mathrm{D} v=$ $\left(\mathrm{D}_{1} v, \ldots, \mathrm{D}_{n} v\right)$ is its distributional gradient. A vector-valued function $v: U \rightarrow \mathbb{R}^{m}$ is $B V\left(U ; \mathbb{R}^{m}\right)$ if $v_{j} \in B V(U)$ for every $j=1, \ldots, m$.

A $\mathcal{L}^{n}$-measurable bounded set $E \subset \mathbb{R}^{n}$ is a set of finite perimeter if $\chi_{E}$ is a function of bounded variation. The reduced boundary of $E$, denoted by $\partial^{*} E$, is the set of points $x \in \operatorname{supp}\left|\mathrm{D} \chi_{E}\right|$ such that the limit $\nu_{E}(x):=\lim _{\varrho \rightarrow 0^{+}} \frac{\mathrm{D} \chi_{E}\left(B_{\varrho}(x)\right)}{\left|\mathrm{D} \chi_{E}\right|\left(B_{\varrho}(x)\right)}$ exists and satisfies $\left|\nu_{E}(x)\right|=1$. The reduced boundary is countably $\left(\mathcal{H}^{n-1}, n-1\right)$ rectifiable, and the function $\nu_{E}$ is called generalised inner normal to $E$.

The space of functions of bounded deformation on $U$ is

$$
B D(U):=\left\{v \in L^{1}\left(U ; \mathbb{R}^{n}\right): \mathrm{E} v \in \mathcal{M}_{b}\left(U ; \mathbb{M}_{\text {sym }}^{n \times n}\right)\right\},
$$

where $\mathrm{E} v$ is the distributional symmetric gradient of $v$. It is well known (see [7, 58]) that for $v \in B D(U)$, the jump set $J_{v}$, defined as the set of points $x \in U$ where $v$ has two different one sided Lebesgue limits $v^{+}(x)$ and $v^{-}(x)$ with respect to a suitable direction $\nu_{v}(x) \in \mathbb{S}^{n-1}$, is countably $\left(\mathcal{H}^{n-1}, n-1\right)$ rectifiable (see, e.g. [38, 3.2.14]), and that

$$
\mathrm{E} v=\mathrm{E}^{a} v+\mathrm{E}^{c} v+\mathrm{E}^{j} v
$$

where $\mathrm{E}^{a} v$ is absolutely continuous with respect to $\mathcal{L}^{n}, \mathrm{E}^{c} v$ is singular with respect to $\mathcal{L}^{n}$ and such that $\left|\mathrm{E}^{c} v\right|(B)=0$ if $\mathcal{H}^{n-1}(B)<\infty$, while

$$
\mathrm{E}^{j} v=[v] \odot \nu_{v} \mathcal{H}^{n-1}\left\llcorner J_{v} .\right.
$$

In the above expression of $\mathrm{E}^{j} v,[v]$ denotes the jump of $v$ at any $x \in J_{v}$ and is defined by $[v](x):=\left(v^{+}-v^{-}\right)(x)$, the symbols $\odot$ and $L$ stands for the symmetric tensor product and the restriction of a measure to a set, respectively. Since $|a \odot b| \geq|a||b| / \sqrt{2}$ for every $a, b$ in $\mathbb{R}^{n}$, it holds $[v] \in L^{1}\left(J_{v} ; \mathbb{R}^{n}\right)$. The density of $\mathrm{E}^{a} v$ with respect to $\mathcal{L}^{n}$ is denoted by $e(v)$, and we have that (see [7, Theorem 4.3]) for $\mathcal{L}^{n}$-a.e. $x \in U$

$$
\text { ap } \lim _{y \rightarrow x} \frac{(v(y)-v(x)-e(v)(x)(y-x)) \cdot(y-x)}{|y-x|^{2}}=0 .
$$

The space $S B D(U)$ is the subspace of all functions $v \in B D(U)$ such that $\mathrm{E}^{c} v=0$, while for $p \in(1, \infty)$

$$
S B D^{p}(U):=\left\{v \in S B D(U): e(v) \in L^{p}\left(U ; \mathbb{M}_{s y m}^{n \times n}\right), \mathcal{H}^{n-1}\left(J_{v}\right)<\infty\right\} .
$$

Analogous properties hold for $B V$, as the countable rectifiability of the jump set and the decomposition of $\mathrm{D} v$, and the spaces $S B V\left(U ; \mathbb{R}^{m}\right)$ and $S B V^{p}\left(U ; \mathbb{R}^{m}\right)$ are defined similarly, with $\nabla v$, the density of $\mathrm{D}^{a} v$ with respect to $\mathcal{L}^{n}$, in place of $e(v)$.

We now recall some slicing properties of $S B D$ that will be useful in Theorem 2.9. As general notation, fixed $\xi \in \mathbb{S}^{n-1}:=\left\{\xi \in \mathbb{R}^{n}:|\xi|=1\right\}$, for any $y \in \mathbb{R}^{n}$ and $B \subset \mathbb{R}^{n}$ let

$$
\Pi^{\xi}:=\left\{y \in \mathbb{R}^{n}: y \cdot \xi=0\right\}, \quad B_{y}^{\xi}:=\{t \in \mathbb{R}: y+t \xi \in B\},
$$

and for every function $v: B \rightarrow \mathbb{R}^{n}$ and $t \in B_{y}^{\xi}$ let

$$
v_{y}^{\xi}(t):=v(y+t \xi), \quad \widehat{v}_{y}^{\xi}(t):=v_{y}^{\xi}(t) \cdot \xi
$$

The following proposition collects some results from [7] (see Propositions 3.2, 4.7, and Theorem 4.5 therein).

Proposition 2.3. Let $v \in L^{1}\left(U ; \mathbb{R}^{n}\right)$ and $e_{1}, \ldots, e_{n}$ be a basis of $\mathbb{R}^{n}$. Then $v \in B D(U)$ [resp. $S B D(U)]$ if and only if for every $\xi=e_{i}+e_{j}, 1 \leq i, j \leq n$

$$
\begin{gathered}
\widehat{v}_{y}^{\xi} \in B V\left(U_{y}^{\xi}\right)\left[\text { resp. } S B V\left(U_{y}^{\xi}\right)\right] \quad \text { for } \mathcal{H}^{n-1} \text {-a.e. } y \in \Pi^{\xi}, \\
\int_{\Pi \xi}\left|\mathrm{D} \widehat{v}_{y}^{\xi}\right|\left(U_{y}^{\xi}\right) \mathrm{d} \mathcal{H}^{n-1}(y)<+\infty .
\end{gathered}
$$


Moreover, let $v \in B D(U), \xi \in \mathbb{S}^{n-1}$ and $J_{v}^{\xi}:=\left\{x \in J_{v}:[v] \cdot \xi \neq 0\right\}$ (it holds that $\mathcal{H}^{n-1}\left(J_{v} \backslash J_{v}^{\xi}\right)=0$ for $\mathcal{H}^{n-1}$-a.e. $\left.\xi \in \mathbb{S}^{n-1}\right)$. Then

$$
\mathrm{E}^{j} v \xi \cdot \xi=\int_{\Pi^{\xi}} \int_{\widehat{v}_{\widehat{v}_{y}^{\xi}}}\left[\widehat{v}_{y}^{\xi}\right] \mathrm{d} t \mathrm{~d} \mathcal{H}^{n-1}(y), \quad\left|\mathrm{E}^{j} v \xi \cdot \xi\right|(U)=\int_{\Pi^{\xi} \widehat{J}_{\widehat{v}_{y}^{\xi}}}\left|\left[\widehat{v}_{y}^{\xi}\right]\right| \mathrm{d} t \mathrm{~d} \mathcal{H}^{n-1}(y),
$$

and for $\mathcal{H}^{n-1}$-a.e. $y \in \Pi^{\xi}$

$$
\begin{gathered}
e(v)_{y}^{\xi} \xi \cdot \xi=\nabla \widehat{v}_{y}^{\xi} \quad \mathcal{L}^{1} \text {-a.e. on } U_{y}^{\xi}, \\
\left(J_{v}^{\xi}\right)_{y}^{\xi}=J_{\widehat{v}_{y}^{\xi}} \quad \text { and } \quad v^{ \pm}(y+t \xi) \cdot \xi=\left(\widehat{v}_{y}^{\xi}\right)^{ \pm}(t) \quad \text { for } t \in\left(J_{v}\right)_{y}^{\xi},
\end{gathered}
$$

where the normals to $J_{v}$ and $J_{\widehat{v_{y}^{\xi}}}$ are oriented so that $\xi \cdot \nu_{v} \geq 0$ and $\nu_{\widehat{v}_{y}^{\xi}}=1$.

For more details on the spaces $B V, S B V$ and $B D, S B D$ we refer to [8] and to [7, 14, 11, 58, respectively.

$G B D$ functions. The space $G B D$ of generalised functions of bounded deformation has been introduced in [34] (to which we refer for a general treatment) and it is defined by slicing as follows.

Definition 2.4 ([34]). Let $\Omega \subset \mathbb{R}^{n}$ be bounded and open, and $v: \Omega \rightarrow \mathbb{R}^{n}$ be $\mathcal{L}^{n_{-}}$ measurable. Then $v \in G B D(\Omega)$ if there exists $\lambda_{v} \in \mathcal{M}_{b}^{+}(\Omega)$ such that the following equivalent conditions hold for every $\xi \in \mathbb{S}^{n-1}$ :

(a) for every $\tau \in C^{1}(\mathbb{R})$ with $-\frac{1}{2} \leq \tau \leq \frac{1}{2}$ and $0 \leq \tau^{\prime} \leq 1$, the partial derivative $\mathrm{D}_{\xi}(\tau(v \cdot \xi))=\mathrm{D}(\tau(v \cdot \xi)) \cdot \xi$ belongs to $\mathcal{M}_{b}(\Omega)$, and for every Borel set $B \subset \Omega$

$$
\left|\mathrm{D}_{\xi}(\tau(v \cdot \xi))\right|(B) \leq \lambda_{v}(B) ;
$$

(b) $\widehat{v}_{y}^{\xi} \in B V_{\text {loc }}\left(\Omega_{y}^{\xi}\right)$ for $\mathcal{H}^{n-1}$-a.e. $y \in \Pi^{\xi}$, and for every Borel set $B \subset \Omega$

$$
\int_{\Pi_{\xi}}\left(\left|\mathrm{D} \widehat{v}_{y}^{\xi}\right|\left(B_{y}^{\xi} \backslash J_{\widehat{v}_{y}^{\xi}}^{1}\right)+\mathcal{H}^{0}\left(B_{y}^{\xi} \cap J_{\widehat{v}_{y}^{\xi}}^{1}\right)\right) \mathrm{d} \mathcal{H}^{n-1}(y) \leq \lambda_{v}(B),
$$

where $J_{\widehat{v}_{y}^{\xi}}^{1}:=\left\{t \in J_{\widehat{v}_{y}^{\xi}}:\left|\left[\widehat{v}_{y}^{\xi}\right]\right|(t) \geq 1\right\}$.

The function $v$ belongs to $G S B D(\Omega)$ if $v \in G B D(\Omega)$ and $\widehat{v}_{y}^{\xi} \in S B V_{\text {loc }}\left(\Omega_{y}^{\xi}\right)$ for every $\xi \in \mathbb{S}^{n-1}$ and for $\mathcal{H}^{n-1}$-a.e. $y \in \Pi^{\xi}$.

$G B D(\Omega)$ and $G S B D(\Omega)$ are vector spaces, as stated in [34, Remark 4.6], and one has the inclusions $B D(\Omega) \subset G B D(\Omega), S B D(\Omega) \subset G S B D(\Omega)$, which are in general strict (see [34. Remark 4.5 and Example 12.3]). Every $v \in G B D(\Omega)$ has an approximate symmetric gradient $e(v) \in L^{1}\left(\Omega ; \mathbb{M}_{\text {sym }}^{n \times n}\right)$, still characterised by (2.3) and (2.6a), and the approximate jump set $J_{v}$ is still countably $\left(\mathcal{H}^{n-1}, n-1\right)$-rectifiable $(c f$. [34, Theorem 6.2]) and can be reconstructed by (2.6b) (see [34, Theorem 8.1]).

First order differential operators $\mathbb{A}$ and functions of bounded $\mathbb{A}$-variation. In this paragraph we recall recent results from [17, 46, 47], starting from the notions of $\mathbb{R}$ - and $\mathbb{C}$-ellipticity for operators $\mathbb{A}$ of the form $(1.3)$, introduced in Section 1. Such an operator can be seen as $\mathbb{A} u=A(\mathrm{E} u)$, for $A$ endomorphism on $\mathbb{M}_{\text {sym }}^{n \times n}$, as in (1.4).

First (see [17, Theorem 2.6]) $\mathbb{A}$ is $\mathbb{C}$-elliptic if and only if the kernel of $\mathbb{A}$, defined by

$$
N(\mathbb{A})=\left\{v \in \mathcal{D}^{\prime}\left(\mathbb{R}^{n} ; \mathbb{R}^{n}\right): \mathbb{A} v \equiv 0\right\},
$$

is finite dimensional and contained in the space of polynomials of degree less than $l=$ $l(\mathbb{A}) \in \mathbb{N}$. 
Remark 2.5. For the symmetrised gradient $\mathbb{A} v=\mathrm{E} v=\frac{1}{2}\left(\nabla v+\nabla v^{T}\right)$, we have $N(\mathrm{E})=$ $\left\{x \mapsto M x+b: M \in \mathbb{M}^{n \times n}, M=-M^{T}, b \in \mathbb{R}^{n}\right\}$. For $\mathbb{A} v=\mathrm{E}_{D} v=\mathrm{E} v-\frac{1}{n}(\operatorname{div} v) \operatorname{Id}_{n}$, if $n \geq 3$ this operator is $\mathbb{C}$-elliptic with

$$
N\left(\mathrm{E}_{D}\right)=\left\{x \mapsto M x+b+\left(2(a \cdot x) x-|x|^{2} a\right): M \in \mathbb{M}^{n \times n}, M=-M^{T}, a, b \in \mathbb{R}^{n}\right\},
$$

while, if $n=2, \mathrm{E}_{D}$ is only $\mathbb{R}$-elliptic and $N\left(\mathrm{E}_{D}\right)$ consists of the holomorphic functions, with the identification $\mathbb{C} \cong \mathbb{R}^{2}$. (The elements of $N\left(\mathrm{E}_{D}\right)$ are usually called conformal Killing vectors, see [32, 44].)

By [17, Lemma 2.3] if $\mathbb{A}$ is $\mathbb{R}$-elliptic there exist $0<\kappa_{1}<\kappa_{2}<\infty$ such that

$$
\kappa_{1}|w||z| \leq\left|w \otimes_{\mathbb{A}} z\right| \leq \kappa_{2}|w||z| \text { for all } w, z \in \mathbb{R}^{n} .
$$

For every open domain $U \subset \mathbb{R}^{n}$, the total $\mathbb{A}$-variation of $v \in L_{\text {loc }}^{1}\left(U ; \mathbb{R}^{n}\right)$ is (notice that $\mathbb{A}$ is symmetric)

$$
|\mathbb{A} v|(U):=\sup \left\{\int_{U} v \cdot \mathbb{A} \varphi \mathrm{d} x: \varphi \in C_{c}^{1}\left(U ; \mathbb{R}^{n}\right),|\varphi| \leq 1\right\} .
$$

A function $v \in L^{1}\left(U ; \mathbb{R}^{n}\right)$ is of bounded $\mathbb{A}$-variation if $|\mathbb{A} v|(U)<\infty$ and we denote

$$
B V^{\mathbb{A}}(U):=\left\{v \in L^{1}\left(U ; \mathbb{R}^{n}\right): \mathbb{A} v \in \mathcal{M}_{b}\left(U ; \mathbb{M}_{\text {sym }}^{n \times n}\right)\right\} .
$$

The following proposition collects [59, Theorem 1.3] (see also [46, Theorem 1.1]), [46, Proposition 4.2 and Lemma 5.8], [47, Proposition 2.5], and [44, Theorem 3].

Proposition 2.6. Let $U$ be bounded and star-shaped with respect to a ball (that is starshaped with respect to each point of a ball $B \subset U$ ). If $\mathbb{A}$ is $\mathbb{C}$-elliptic then there exist a constant $C>0$ such that

$$
\|v\|_{L^{1^{*}}\left(U ; \mathbb{R}^{n}\right)} \leq C\|\mathbb{A} v\|_{L^{1}\left(U ; \mathbb{M}_{s y m}^{n \times n}\right)}
$$

for every $v \in C_{c}^{1}\left(U ; \mathbb{R}^{n}\right)$, and (denoting by $\hookrightarrow$ and $\hookrightarrow \hookrightarrow$ continuous and compact embeddings, respectively)

$$
\begin{aligned}
& B V^{\mathbb{A}}(U) \hookrightarrow L^{1^{*}}\left(U ; \mathbb{R}^{n}\right) \\
& B V^{\mathbb{A}}(U) \hookrightarrow \hookrightarrow L^{p}\left(U ; \mathbb{R}^{n}\right) \text { for every } p \in\left[1,1^{*}\right) .
\end{aligned}
$$

If $\mathbb{A}$ is $\mathbb{R}$-elliptic then for every $p \in\left[1,1^{*}\right)$ there exist $C_{p}>0$ such that

$$
\|v\|_{L^{p}\left(U ; \mathbb{R}^{n}\right)} \leq C_{p}\|\mathbb{A} v\|_{L^{1}\left(U ; \mathbb{M}_{s y m}^{n \times n}\right)}
$$

for every $v \in C_{c}^{1}\left(U ; \mathbb{R}^{n}\right)$. Moreover, if $\mathbb{A}$ is $\mathbb{C}$-elliptic then there is $C>0$, depending only on $n$, such that for every $v \in B V^{\mathbb{A}}(U)$

$$
\left\|v-\pi_{U} v\right\|_{L^{1^{*}}(U)} \leq C|\mathbb{A} v|(U),
$$

for a suitable $\pi_{U} v \in N(\mathbb{A})$. If $n=2$, then for every $p \in\left[1,1^{*}\right)$ there exists $C>0$ depending only on $p$, such that it holds

$$
\left\|v-\pi_{U} v\right\|_{L^{p}(U)} \leq C \operatorname{diam}(U)^{1-n+\frac{n}{p}}\left|\mathrm{E}_{D} v\right|(U),
$$

for some $\pi_{U} v \in N\left(\mathrm{E}_{D}\right)$, namely $\pi_{U} v$ is holomorphic (see Remark 2.7).

Remark 2.7. In [59] it is proven that $(2.10)$ is equivalent to the fact that $\mathbb{A}$ is $\mathbb{R}$-elliptic and cancelling, a weaker property than $\mathbb{C}$-ellipticity. For $n=2$, we have that $\mathrm{E}_{D}$ is only $\mathbb{R}$-elliptic but not cancelling, so only $(2.12)$ holds, and $N\left(\mathrm{E}_{D}\right)$ can be identified with the space of holomorphic functions (see [46, Example $2.4 \mathrm{c}$ )]). 
Remark 2.8. The estimates 2.13 and 2.14 may be extended to any connected set $U$ finite union of sets $U_{i}$ which are bounded and star-shaped with respect to a ball. Indeed, since $N(\mathbb{A})$ is made of polynomials and due to $(2.13)$, one can find $\pi_{U} \in N(\mathbb{A})$ such that $\left\|\pi_{U_{i}} v-\pi_{U} v\right\|_{L^{1^{*}}(U)} \leq C|\mathbb{A} v|(U)$ for any $i$. This is true, for $\pi_{U_{j}} v$ in place of $\pi_{U} v$, for any $U_{i}, U_{j}$ with $\mathcal{L}^{n}\left(U_{i} \cap U_{j}\right)>0$, by rigidity of polynomials, and it is extended to a finite union. As for (2.14), see [44, comment before Theorem 3]. In particular, one sees that (2.13) and 2.14 hold if $U$ is a connected Lipschitz domain.

We prove below the main result of the section.

Theorem 2.9. Let $U \subset \mathbb{R}^{n}$ be an open bounded domain. If $\mathbb{A}$ as in 1.3 (i.e., $\mathbb{A}$ symmetric) is $\mathbb{C}$-elliptic, then

$$
G B D(U) \cap B V^{\mathbb{A}}(U)=B D(U)
$$

and

$$
G S B D(U) \cap B V^{\mathbb{A}}(U)=S B D(U) .
$$

Proof. By 2.4 , we have that from 2.15a one gets 2.15b). It is also immediate that $B D(U) \subset G B D(U) \cap B V^{\mathbb{A}}(U)$, being $\mathbb{A}$ symmetric. In order to show the opposite inclusion, let us fix $u \in G B D(U) \cap B V^{\mathbb{A}}(U)$ and first prove (in the spirit of the blow up technique [41) that

$$
\frac{\mathrm{d}|\mathbb{A} u|}{\mathrm{d} \mathcal{H}^{n-1}\left\llcorner J_{u}\right.} \geq\left|[u] \otimes_{\mathbb{A}} \nu_{u}\right| \quad \mathcal{H}^{n-1} \text {-a.e. in } J_{u} .
$$

Since $J_{u}$ is countably rectifiable, so that $\mathcal{H}^{n-1}\left\llcorner J_{u}\right.$ is $\sigma$-finite, and $\mathbb{A} u \in \mathcal{M}_{b}\left(U ; \mathbb{M}_{\text {sym }}^{n \times n}\right)$, the Radon-Nikodym derivative of $|\mathbb{A} u|$ with respect to $\mathcal{H}^{n-1}\left\llcorner J_{u}\right.$ exists (more precisely, it is the function $\theta \in L^{1}\left(J_{u}\right)$ such that $\left|\mathbb{A} u^{a}\right|=\theta \mathcal{H}^{n-1}\left\llcorner J_{u}\right.$, where $\mathbb{A} u=\mathbb{A} u^{a}+\mathbb{A} u^{s}$, for $\mathbb{A} u^{a} \ll \mathcal{H}^{n-1}\left\llcorner J_{u}, \mathbb{A} u^{s} \perp \mathcal{H}^{n-1}\left\llcorner J_{u}\right)\right.$. Moreover, it may be computed explicitly by (see e.g. [8, Theorems 1.28 and 2.83]):

$$
\frac{\mathrm{d}|\mathbb{A} u|}{\mathrm{d} \mathcal{H}^{n-1}\left\llcorner J_{u}\right.}(x)=\lim _{\varrho \rightarrow 0} \frac{|\mathbb{A} u|\left(B_{\varrho}(x)\right)}{\mathcal{H}^{n-1}\left(J_{u} \cap B_{\varrho}(x)\right)} \quad \text { for } \mathcal{H}^{n-1} \text {-a.e. } x \in J_{u} .
$$

For $\mathcal{H}^{n-1}$-a.e. $x \in J_{u}$, we have also that

$$
\lim _{\varrho \rightarrow 0} \frac{\mathcal{H}^{n-1}\left(J_{u} \cap B_{\varrho}(x)\right)}{\omega_{n-1} \varrho^{n-1}}=1
$$

for $\omega_{n-1}$ the $n$-1-dimensional measure of the unit ball in $\mathbb{R}^{n-1}$, and that, if we introduce $u_{\varrho, x}(y):=u(x+\varrho y): B_{1}(0) \rightarrow \mathbb{R}^{n}$, then (denoting $B:=B_{1}(0)$ )

$$
\lim _{\varrho \rightarrow 0^{+}} u_{\varrho, x} \rightarrow u_{0}:=u^{+}(x) \chi_{B^{+}}+u^{-}(x) \chi_{B^{-}} \text {in } \mathcal{L}^{n} \text {-measure in } B,
$$

where $u^{ \pm}(x) \in \mathbb{R}^{n}$ are the Lebesgue limits at $x$ on the two sides of $J_{u}$ with respect to $\nu_{u}(x)$, and $B^{ \pm}:=B \cap\left\{y \in \mathbb{R}^{n}:(y-x) \cdot \nu_{u}(x) \in \mathbb{R}^{ \pm}\right\}$(see also e.g. 34, Theorem 6.2, below (6.4)]). Let us fix $x$ such that these three conditions hold, and denote $u_{\varrho} \equiv u_{\varrho, x}$.

Since the derivative in 2.17) exists finite, by 2.18 and the fact that

$$
\left|\mathbb{A} u_{\varrho}\right|(B)=\frac{|\mathbb{A} u|\left(B_{\varrho}(x)\right)}{\varrho^{n-1}}
$$

we obtain that there exists $C>0$ independent of $\varrho$ such that

$$
\left|\mathbb{A} u_{\varrho}\right|(B) \leq C \text {. }
$$

By the embeddings 2.11 we get that $\left\|u_{\varrho}\right\|_{L^{1}(B)}=\varrho^{-n}\|u\|_{L^{1}\left(B_{\varrho}(x)\right)}<\infty$, so that $u_{\varrho} \in$ $B V^{\mathbb{A}}(B)$ for any $\varrho>0$ and 2.13 , 2.20 imply

$$
\left\|u_{\varrho}-\pi_{\varrho}\right\|_{L^{1^{*}(B)}} \leq C,
$$


where $\pi_{\varrho}:=\pi_{B} u_{\varrho}$. This gives that $\left(u_{\varrho}-\pi_{\varrho}\right)_{\varrho}$ is bounded in $B V^{\mathbb{A}}(B)$. Then, by (2.11), up to a (not relabelled) subsequence, $u_{\varrho}-\pi_{\varrho} \rightarrow \tilde{v} \in \mathbb{R}^{n}$ a.e. in $B$. Recalling (2.19), $\pi_{\varrho}$ belong to the finite dimensional space of polynomials $N(\mathbb{A})$ of degree less than $l(\mathbb{A}) \in \mathbb{N}$ (being $\mathbb{A}$ elliptic, cf. before Remark 2.5) and converge $\mathcal{L}^{n}$-a.e. in $B$. Therefore $\pi_{\varrho}$ converge uniformly to a suitable polynomial $\pi_{0}$ (indeed, if $\left\|\pi_{\varrho}\right\| \rightarrow \infty$, for any norm on the finite dimensional space of polynomials of degree less than $l(\mathbb{A})$, then $\frac{\pi_{\varrho}}{\left\|\pi_{\varrho}\right\|}$ converges to a polynomial of degree less than $l(\mathbb{A})$, so $\left|\pi_{\varrho}\right|$ converges to $+\infty$ up to a $\mathcal{L}^{n}$-negligible set).

By difference we obtain that the convergence in $(2.19)$ is strong in $L^{1}\left(B ; \mathbb{R}^{n}\right)$, passing to a suitable subsequence $\varrho_{k}$. Looking at the definition of $|\mathbb{A} u|$ in $(2.9)$, we deduce immediately the lower semicontinuity with respect to $L^{1}$-convergence of $u_{\varrho}$, so by 2.17$), 2.18$ )

$$
\begin{aligned}
\left|\mathbb{A} u_{0}\right|(B) & \leq \liminf _{k \rightarrow \infty}\left|\mathbb{A} u_{\varrho_{k}}\right|(B)=\liminf _{k \rightarrow \infty} \frac{|\mathbb{A} u|\left(B_{\varrho_{k}}(x)\right)}{\left(\varrho_{k}\right)^{n-1}} \\
& =\lim _{\varrho \rightarrow 0} \frac{|\mathbb{A} u|\left(B_{\varrho}(x)\right)}{\varrho^{n-1}}=\omega_{n-1} \frac{\mathrm{d}|\mathbb{A} u|}{\mathrm{d} \mathcal{H}^{n-1}\left\llcorner J_{u}\right.}(x) .
\end{aligned}
$$

By the special form of $u_{0}$ (see 2.19), we have directly that

$$
\left|\mathbb{A} u_{0}\right|(B)=\omega_{n-1}\left|[u](x) \otimes_{\mathbb{A}} \nu_{u}(x)\right| .
$$

This proves the claim 2.16).

Combining 2.16 with 2.8 (recall that $\mathbb{A} u$ has bounded variation) we obtain that

$$
[u] \in L^{1}\left(J_{u} ; \mathbb{R}^{n}\right),
$$

for $[u]=u^{+}-u^{-}$, where $u^{ \pm}$are the Lebesgue limits in the sense of $G B D$, cf. Definition 2.2

It is now possible to fill the gap between the slicing conditions (2.7) for $G(S) B D$ and the characterisation of $(S) B D$ functions $(2.4)$, by the area formula for rectifiable sets (see e.g. [56, (12.4) in Section 12]). Since $J_{u}$ is countably $\left(\mathcal{H}^{n-1}, n-1\right)$-rectifiable and $\nu_{u} \cdot \xi$ is the Jacobian of the projection $p_{\xi}: J_{u} \rightarrow \Pi^{\xi}$ (we consider $\nu_{u} \cdot \xi \geq 0$ ) we obtain for any $\xi \in \mathbb{S}^{n-1}$

$$
\int_{J_{u}^{\xi}}|[u] \cdot \xi|\left(\nu_{u} \cdot \xi\right) \mathrm{d} \mathcal{H}^{n-1}=\int_{\Pi^{\xi}} \sum_{t \in\left(J_{u}^{\xi}\right)_{y}^{\xi}}|[u](y+t \xi) \cdot \xi| \mathrm{d} \mathcal{H}^{n-1}=\int_{\Pi^{\xi}} \sum_{t \in J_{\widehat{u}_{y}^{\xi}}}\left|\left[\widehat{u}_{y}^{\xi}\right]\right|(t) \mathrm{d} \mathcal{H}^{n-1},
$$

recalling that (2.6b) holds also for $u \in G B D(U)$. Employing (2.5) and 2.7) in Definition 2.4 (now $\widehat{u}_{y}^{\xi} \in S B V_{\text {loc }}\left(U_{y}^{\xi}\right)$ for $\mathcal{H}^{n-1}$-a.e. $\xi$ if $u \in G S B D(U)$ ), and the fact that 2.6a) holds both in $(S) B D$ and $G(S) B D$, we get (2.4) and then $u \in B D(U)$. This concludes the proof.

Remark 2.10. For $n=2$ and $\mathbb{A}=\mathrm{E}_{D}$ we are not able to deduce that $G B D \cap B V^{\mathrm{E}_{D}}=B D$ as above, the issue being property (2.16) (if this was true, then we would conclude by using (2.8)). Indeed, in this case $N\left(\mathrm{E}_{D}\right)$ consists of the holomorphic functions (with the identification $\mathbb{C} \cong \mathbb{R}^{2}$ ); employing (2.14) instead of (2.13) we get (2.21) with any fixed $p \in\left[1,1^{*}\right)$ in place of $1^{*}$, where $\pi_{\varrho}$ is holomorphic. Now the problem is that it is not true that the $\mathcal{L}^{n}$-a.e. convergence of $\pi_{\varrho}$ to $\pi_{0}:=u_{0}-\tilde{v}$ takes place also in $L^{1}$ : in general, the convergence is locally uniform just on an open dense subset of $B_{1}(0)$ (by Osgood's theorem [55]).

Corollary 2.11. If $\mathbb{A}$ is an operator as in Theorem 2.9 and $u \in G B D(U) \cap B V^{\mathbb{A}}(U)$, then

$$
\mathbb{A} u\left\llcorner J_{u}=[u] \otimes_{\mathbb{A}} \nu_{u} \mathcal{H}^{n-1}\left\llcorner J_{u},\right.\right.
$$

applying the operator $\mathbb{A}$ to 2.2 . 


\section{3. $\Gamma$-lim inf INEQUALITY}

Let us fix a sequence $\varepsilon_{k}$ and denote by $D_{k}$ the functionals $D_{\varepsilon_{k}}$, with analogous notation for all the quantities depending on $\varepsilon$. We consider an open bounded domain $\Omega^{\prime} \subset \mathbb{R}^{n}$ such that $\Omega \subset \Omega^{\prime}$ and $\Omega^{\prime} \cap \partial \Omega=\partial_{D} \Omega$ and set, for each $u, v$ defined in $\Omega$, their extensions

$$
\tilde{u}:=\left\{\begin{array}{ll}
u & \text { in } \Omega, \\
u_{0} & \text { in } \Omega^{\prime} \backslash \Omega,
\end{array} \quad \tilde{v}:= \begin{cases}v & \text { in } \Omega, \\
1 & \text { in } \Omega^{\prime} \backslash \Omega,\end{cases}\right.
$$

Then we have that

$$
\begin{aligned}
& D_{k}^{\Omega^{\prime}}(\tilde{u}, \tilde{v})-D_{k}^{\Omega}(u, v)=D^{\Omega^{\prime}}(\tilde{u}, v)-D^{\Omega}(u, v) \\
& \quad=\int_{\Omega^{\prime} \backslash \Omega}\left[\left(1+\varepsilon_{k}^{p-1}\right) f_{p}\left(\mathbb{A} u_{0}\right)+\left(1+\eta_{\varepsilon_{k}}\right) f_{p}\left(e\left(u_{0}\right)-\mathbb{A} u_{0}\right)\right] \mathrm{d} x,
\end{aligned}
$$

where $D_{k}^{\Omega}, D^{\Omega}$ and $D_{k}^{\Omega^{\prime}}, D^{\Omega^{\prime}}$ are the functionals $D_{k}$ and $D$ with the integrals evaluated on $\Omega$ and $\Omega^{\prime}$. Then it is enough to argue in the enlarged domain $\Omega^{\prime}$. We denote $\tilde{D}_{k}:=D_{k}^{\Omega^{\prime}}$, $\tilde{D}:=D^{\Omega^{\prime}}$

First we prove that for given sequences $u_{k}, v_{k}$ converging in $\mathcal{L}^{n}$-measure to some $u: \Omega \rightarrow$ $\mathbb{R}^{n}, v: \Omega \rightarrow \mathbb{R}$ measurable, such that $D_{k}\left(u_{k}, v_{k}\right)<\infty$, that is

$$
\tilde{D}_{k}\left(\tilde{u}_{k}, \tilde{v}_{k}\right)<\infty,
$$

(we may assume without loss of generality that $D\left(u_{k}, v_{k}\right)$, and then $\tilde{D}\left(\tilde{u}_{k}, \tilde{v}_{k}\right)$, converges to some finite limit) we have

$$
\tilde{u} \in G S B D^{p}\left(\Omega^{\prime}\right) \cap B V^{\mathbb{A}}\left(\Omega^{\prime}\right) \text { and } \quad \tilde{v}=1 \text { a.e. in } \Omega^{\prime} .
$$

Since $\tilde{D}_{k}\left(\tilde{u}_{k}, \tilde{v}_{k}\right) \geq \int_{\Omega} \frac{\psi\left(\tilde{v}_{k}\right)}{\varepsilon_{k}} \mathrm{~d} x$ and $\psi$ is decreasing with $\psi(1)=0$, we get readily that $\tilde{v}=1$ a.e. in $\Omega^{\prime}$ and $\tilde{v}_{k} \rightarrow 1$ in $\mathcal{L}^{n}$-measure. As for $u$, recalling the assumptions on $f_{p}$, we have that for any $\lambda \in[0,1)$

$$
\begin{aligned}
& \tilde{D}_{k}\left(\tilde{u}_{k}, \tilde{v}_{k}\right) \geq C_{f_{p}} \int_{\Omega^{\prime}}\left[\lambda\left|A\left(e\left(\tilde{u}_{k}\right)\right)\right|^{p} \chi_{\left\{\tilde{v}_{k} \geq \lambda\right\}}+\varepsilon_{k}^{p-1}\left|A\left(e\left(\tilde{u}_{k}\right)\right)\right|^{p} \chi_{\left\{\tilde{v}_{k} \leq \lambda\right\}}\right] \mathrm{d} x \\
& \quad+\frac{\psi(\lambda)}{\varepsilon_{k}} \mathcal{L}^{n}\left(\left\{\tilde{v}_{k} \leq \lambda\right\}\right)-C_{f_{p}} \mathcal{L}^{n}\left(\Omega^{\prime}\right) \\
& \geq \widetilde{C}_{f_{p}, p}\left[\lambda \int_{\left\{\tilde{v}_{k} \geq \lambda\right\}}\left|A\left(e\left(\tilde{u}_{k}\right)\right)\right|^{p} \mathrm{~d} x+p^{\frac{1}{p}}\left(p^{\prime}\right)^{\frac{1}{p^{\prime}}} \psi(\lambda)^{\frac{1}{p^{\prime}}} \int_{\left\{\tilde{v}_{k} \leq \lambda\right\}}\left|A\left(e\left(\tilde{u}_{k}\right)\right)\right| \mathrm{d} x\right]-C_{f_{p}} \mathcal{L}^{n}\left(\Omega^{\prime}\right) \\
& \geq \widetilde{C} \int_{\Omega^{\prime}}\left|A\left(e\left(\tilde{u}_{k}\right)\right)\right| \mathrm{d} x-\widehat{C},
\end{aligned}
$$

for suitable $\widetilde{C}, \widehat{C}$ depending on $f_{p}, p, \psi, \mathcal{L}^{n}(\Omega)$, and $\lambda$. Notice that we have employed the operator $A$ in $(1.4)$ to underline the dependence on the absolutely continuous part $e\left(u_{k}\right)$ and used the Young inequality for the second estimate above.

Since $\tilde{u}_{k} \in L^{1}\left(\Omega^{\prime} ; \mathbb{R}^{n}\right)$, from 2.13$)$ and Remark 2.8 (we may assume here $\Omega^{\prime}$ connected, arguing for each connected component of $\Omega$ ) we get that there are suitable $\pi_{\Omega^{\prime}} \tilde{u}_{k} \in N(\mathbb{A})$ for which

$$
\left\|\tilde{u}_{k}-\pi_{\Omega^{\prime}} \tilde{u}_{k}\right\|_{L^{1^{*}\left(\Omega^{\prime}\right)}} \leq\left\|\mathbb{A}\left(e\left(\tilde{u}_{k}\right)\right)\right\|_{L^{1}\left(\Omega^{\prime}\right)} .
$$

By (3.4) and the compact embedding in (2.11), up to a subsequence the functions $\tilde{u}_{k}-\pi_{\Omega^{\prime}} \tilde{u}_{k}$ converge strongly in $L^{1}\left(\Omega^{\prime} ; \mathbb{R}^{n}\right)$. Since $\tilde{u}_{k}$ converge in measure to $\tilde{u}$, then $\pi_{\Omega^{\prime}} \tilde{u}_{k}$ converge uniformly to a polynomial in $N(\mathbb{A})$ (see the proof of Theorem 2.9), and then, for every $p \in\left[1,1^{*}\right)$, 


$$
\tilde{u}_{k} \rightarrow \tilde{u} \in B V^{\mathbb{A}}\left(\Omega^{\prime}\right) \quad \text { in } L^{p}\left(\Omega^{\prime} ; \mathbb{R}^{n}\right), \quad \mathbb{A} \tilde{u}_{k} \stackrel{*}{\rightarrow} \mathbb{A} \tilde{u} \quad \text { in } \mathcal{M}_{b}\left(\Omega^{\prime} ; \mathbb{M}_{\text {sym }}^{n \times n}\right) .
$$

Let us now prove that $\tilde{u}$ is in $G S B D^{p}\left(\Omega^{\prime}\right)$, employing the two terms depending only on $v$ in $D_{k}$ to estimate the measure of $J_{\tilde{u}}$. Consider the function $\phi(t):=\int_{0}^{t} \psi^{\frac{1}{q^{\prime}}}(s) \mathrm{d} s$ for $t \in[0,1]$. By the Young inequality $\frac{\alpha^{q}}{q}+\frac{\beta^{q^{\prime}}}{q^{\prime}} \geq \alpha \beta$ for

$$
\alpha=\left(\gamma q \varepsilon_{k}^{q-1}\left|\nabla v_{k}\right|^{q}\right)^{1 / q}, \quad \beta=\left(q^{\prime} \psi\left(v_{k}\right) \varepsilon_{k}^{-1}\right)^{1 / q^{\prime}},
$$

we find that for any $\lambda \in(0,1)$

$$
\begin{aligned}
& \tilde{D}_{k}\left(\tilde{u}_{k}, \tilde{v}_{k}\right) \geq \int_{\left\{\tilde{v}_{k}>\lambda\right\}}\left[\frac{\psi\left(\tilde{v}_{k}\right)}{\varepsilon_{k}}+\gamma \varepsilon_{k}^{q-1}\left|\nabla \tilde{v}_{k}\right|^{q}\right] \mathrm{d} x \geq q^{\prime \frac{1}{q^{\prime}}}(\gamma q)^{\frac{1}{q}} \int_{\left\{\tilde{v}_{k}>\lambda\right\}} \psi^{\frac{1}{q^{\prime}}}\left(\tilde{v}_{k}\right)\left|\nabla \tilde{v}_{k}\right| \mathrm{d} x \\
& =q^{\prime \frac{1}{q^{\prime}}(\gamma q)^{\frac{1}{q}}} \int_{\left\{\tilde{v}_{k}>\lambda\right\}}\left|\nabla\left(\phi\left(\tilde{v}_{k}\right)\right)\right| \mathrm{d} x \\
& =q^{\prime \frac{1}{q^{\prime}}}(\gamma q)^{\frac{1}{q}} \int_{\phi(\lambda)}^{\phi(1)} \mathcal{H}^{n-1}\left(\partial^{*}\left\{\phi\left(\tilde{v}_{k}\right)>s\right\}\right) \mathrm{d} s,
\end{aligned}
$$

employing the Coarea formula for $\phi\left(\tilde{v}_{k}\right)$. Therefore, fixed $\lambda \in(0,1)$, for any $\lambda^{\prime} \in(\lambda, 1)$ the Mean Value theorem guarantees the existence of $\tilde{\lambda}_{k} \in\left(\lambda, \lambda^{\prime}\right)$ such that (notice that $\phi$ is strictly increasing)

$$
\begin{aligned}
\mathcal{H}^{n-1} & \left(\partial^{*}\left\{\tilde{v}_{k}>\lambda_{k}\right\}\right)=\mathcal{H}^{n-1}\left(\partial^{*}\left\{\phi\left(\tilde{v}_{k}\right)>\phi\left(\lambda_{k}\right)\right\}\right) \\
& \leq\left(\phi\left(\lambda^{\prime}\right)-\phi(\lambda)\right)^{-1} \int_{\phi(\lambda)}^{\phi\left(\lambda^{\prime}\right)} \mathcal{H}^{n-1}\left(\partial^{*}\left\{\phi\left(\tilde{v}_{k}\right)>s\right\}\right) \mathrm{d} s<C .
\end{aligned}
$$

It follows that the functions $\widehat{u}_{k}:=\tilde{u}_{k} \chi_{\left\{\tilde{v}_{k}>\lambda_{k}\right\}}$ satisfy

$$
\mathrm{E} \widehat{u}_{k}=e\left(\tilde{u}_{k}\right) \chi_{\left\{\tilde{v}_{k}>\lambda_{k}\right\}} \mathcal{L}^{n}+\tilde{u}_{k} \odot \nu_{\partial^{*}\left\{\tilde{v}_{k}>\lambda_{k}\right\}} \mathcal{H}^{n-1}\left\llcorner\partial^{*}\left\{\tilde{v}_{k}>\lambda_{k}\right\} .\right.
$$

Since $\lambda_{k} \geq \lambda>0$, we get a control for $e\left(\tilde{u}_{k}\right)$ in $L^{p}$, and with the estimate above this gives

$$
\int_{\Omega}\left|e\left(\widehat{u}_{k}\right)\right|^{p} \mathrm{~d} x+\mathcal{H}^{n-1}\left(J_{\widehat{u}_{k}}\right) \leq C,
$$

and

$$
\mathcal{L}^{n}\left(\left\{\tilde{u}_{k} \neq \widehat{u}_{k}\right\}\right)=\mathcal{L}^{n}\left(\left\{\tilde{v}_{k} \leq \lambda_{k}\right\}\right) \leq \mathcal{L}^{n}\left(\left\{\tilde{v}_{k} \leq \lambda^{\prime}\right\}\right) \leq \varepsilon_{k} \frac{\tilde{D}_{k}\left(\tilde{u}_{k}, \tilde{v}_{k}\right)}{\mathcal{L}^{n}(\Omega) \psi\left(\lambda^{\prime}\right)} \rightarrow 0
$$

so that

$$
\widehat{u}_{k} \rightarrow \tilde{u} \quad \text { in } \mathcal{L}^{n} \text {-measure in } \Omega^{\prime}
$$

By (3.8) and (3.9) (this latter condition implies that there exists a continuous function $\tilde{\psi}$ diverging to $+\infty$ such that $\int_{\Omega^{\prime}} \tilde{\psi}\left(\tilde{u}_{k}\right) \mathrm{d} x<C<+\infty$ ), we may apply [34, Theorem 11.3] (or we may use the compactness theorem for GSBD [26, Theorem 1.1], since the exceptional set $A$ therein is empty by $(3.9)$ ) to get

$$
\tilde{u} \in G S B D^{p}\left(\Omega^{\prime}\right), \quad e\left(\widehat{u}_{k}\right) \rightarrow e(\tilde{u}) \text { in } L^{p}\left(\Omega^{\prime} ; \mathbb{M}_{\text {sym }}^{n \times n}\right) .
$$

Together with 3.5 this proves the claim (3.3). At this stage Theorem 2.9 implies that

$$
\tilde{u} \in S B D^{p}\left(\Omega^{\prime}\right) .
$$

By the weak convergences 3.10$)$, the fact that $\tilde{v}_{k}$ converge to 1 uniformly up to a set of vanishing measure, and the Ioffe-Olech semicontinuity theorem, see e.g. [19, Theorem 2.3.1], 
we get that for any $\lambda \in(0,1)$ (cf. [39, (4.4) in proof of Theorem 3.3] and 25, (5.4a) in proof of Theorem 5.1])

$$
\begin{aligned}
& \int_{\Omega^{\prime}}\left[f_{p}(A(e(\tilde{u})))+f_{p}(e(\tilde{u})-A(e(\tilde{u})))\right] \mathrm{d} x \\
& \quad \leq \liminf _{k \rightarrow \infty} \int_{\left\{\tilde{v}_{k}>\lambda\right\}}\left[\left(\tilde{v}_{k}+\varepsilon_{k}^{p-1}\right) f_{p}\left(\mathbb{A} \tilde{u}_{k}\right)+\left(\tilde{v}_{k}+\eta_{\varepsilon_{k}}\right) f_{p}\left(e\left(\tilde{u}_{k}\right)-\mathbb{A} \tilde{u}_{k}\right)\right] \mathrm{d} x .
\end{aligned}
$$

As for the Ambrosio-Tortorelli term $\int_{\Omega}\left[\frac{\psi\left(\tilde{v}_{k}\right)}{\varepsilon_{k}}+\gamma \varepsilon_{k}^{q-1}\left|\nabla \tilde{v}_{k}\right|^{q}\right] \mathrm{d} x$ in $\tilde{D}_{k}$, by a standard argument (see e.g. [39, (4.18)], now we argue in the enlarged domain $\Omega^{\prime}$ ) we obtain

$$
\liminf _{k \rightarrow \infty} \mathcal{H}^{n-1}\left(\partial^{*}\left\{\phi\left(\tilde{v}_{k}\right)>s\right\}\right) \geq 2 \mathcal{H}^{n-1}\left(J_{\tilde{u}}\right)=2 \mathcal{H}^{n-1}\left(J_{u} \cup\left(\partial_{D} \Omega \cap\left\{\operatorname{tr}\left(u-u_{0}\right) \neq 0\right\}\right)\right)
$$

for every $s \in(\phi(\lambda), \phi(1))$. Together with (3.7) this gives

$$
\begin{aligned}
& 2\left(q^{\prime}\right)^{1 / q^{\prime}}(\gamma q)^{1 / q}(\phi(1)-\phi(\lambda)) \mathcal{H}^{n-1}\left(J_{u} \cup\left(\partial_{D} \Omega \cap\left\{\operatorname{tr}\left(u-u_{0}\right) \neq 0\right\}\right)\right) \\
& \quad \leq \liminf _{k \rightarrow \infty} \int_{\left\{\tilde{v}_{k}>\lambda\right\}}\left[\frac{\psi\left(\tilde{v}_{k}\right)}{\varepsilon_{k}}+\gamma \varepsilon_{k}^{q-1}\left|\nabla \tilde{v}_{k}\right|^{q}\right] \mathrm{d} x .
\end{aligned}
$$

Let us now estimate the other significant term in the limit by

$$
\int_{\left\{\tilde{v}_{k} \leq \lambda\right\}}\left[\left(\tilde{v}_{k}+\varepsilon_{k}^{p-1}\right) f_{p}\left(\mathbb{A} \tilde{u}_{k}\right)+\frac{\psi\left(\tilde{v}_{k}\right)}{\varepsilon_{k}}\right] \mathrm{d} x \geq p^{\frac{1}{p}}\left(p^{\prime}\right)^{\frac{1}{p^{\prime}}} \psi(\lambda)^{\frac{1}{p^{\prime}}} \int_{\left\{\tilde{v}_{k} \leq \lambda\right\}}\left(f_{p}\right)^{\frac{1}{p}}\left(\mathbb{A} \tilde{u}_{k}\right) \mathrm{d} x,
$$

thanks to the Young inequality. We claim that for any $\lambda \in(0,1)$

$$
\int_{J_{\tilde{u}}}\left(\tilde{f}_{p}\right)^{\frac{1}{p}}\left([\tilde{u}] \otimes_{\mathbb{A}} \nu_{u}\right) \mathrm{d} \mathcal{H}^{n-1} \leq \liminf _{k \rightarrow \infty} \int_{\left\{\tilde{v}_{k} \leq \lambda\right\}}\left(f_{p}\right)^{\frac{1}{p}}\left(\mathbb{A} \tilde{u}_{k}\right) \mathrm{d} x .
$$

Up to a subsequence, that we do not relabel, we may assume that the lim inf above is a limit, so it is enough to prove (3.14) along any further subsequence. Let us introduce the positive measures defined on any $B \subset \Omega^{\prime}$ Borel set by

$$
\mu_{k}(B):=\int_{B \cap\left\{\tilde{v}_{k} \leq \lambda\right\}}\left(f_{p}\right)^{\frac{1}{p}}\left(\mathbb{A} \tilde{u}_{k}\right) \mathrm{d} x, \quad \widehat{\mu}_{k}(B):=\int_{B}\left(\tilde{f}_{p}\right)^{\frac{1}{p}}\left(\mathbb{A} \tilde{u}_{k}\right) \mathrm{d} x .
$$

By (3.5) we get that $\mu_{k}$ and $\widehat{\mu}_{k}$ are equibounded, and then (up to a subsequence)

$$
\mu_{k} \stackrel{*}{\rightarrow} \mu, \quad \widehat{\mu}_{k} \stackrel{*}{\rightarrow} \widehat{\mu} \quad \text { in } \mathcal{M}_{b}^{+}\left(\Omega^{\prime}\right) .
$$

Therefore we want to prove that the Radon-Nikodym derivatives of $\mu$ and $\widehat{\mu}$ satisfy

$$
\frac{\mathrm{d} \mu}{\mathrm{d} \mathcal{H}^{n-1}\left\llcorner J_{\tilde{u}}\right.}=\frac{\mathrm{d} \widehat{\mu}}{\mathrm{d} \mathcal{H}^{n-1}\left\llcorner J_{\tilde{u}}\right.} \geq\left(\tilde{f}_{p}\right)^{\frac{1}{p}}\left([\tilde{u}] \otimes_{\mathbb{A}} \nu_{\tilde{u}}\right) \quad \mathcal{H}^{n-1} \text {-a.e. in } J_{\tilde{u}} .
$$

With (3.15) at disposal, we conclude (3.14) since then

$$
\int_{J_{\tilde{u} \cap B}}\left(\tilde{f}_{p}\right)^{\frac{1}{p}}\left([\tilde{u}] \otimes_{\mathbb{A}} \nu_{\tilde{u}}\right) \mathrm{d} \mathcal{H}^{n-1} \leq \mu(B)
$$

as (positive) measures on $\Omega^{\prime}$, and

$$
\mu\left(\Omega^{\prime}\right) \leq \liminf _{k \rightarrow \infty} \mu_{k}\left(\Omega^{\prime}\right)=\liminf _{k \rightarrow \infty} \int_{\left\{\tilde{v}_{k} \leq \lambda\right\}}\left(f_{p}\right)^{\frac{1}{p}}\left(\mathbb{A} \tilde{u}_{k}\right) \mathrm{d} x .
$$

In order to show (3.15), we argue in the spirit of [39, Proof of (4.6)] (the functions giving the density of elastic energy are there supposed to be quadratic in $e(u)$, we include the 
case where these have $p$-growth and are not $p$-homogeneous, cf. $\left(\overline{H P 2 f_{p}}\right)$ ). Let us define the measures $\zeta_{k} \in \mathcal{M}_{b}^{+}\left(\Omega^{\prime}\right)$ by

$$
\zeta_{k}(B):=D_{k}^{B}\left(\tilde{u}_{k}, \tilde{v}_{k}\right), \quad \text { for } B \subset \Omega^{\prime} \text { Borel, }
$$

where $D_{k}^{B}$ denotes the localisation of $D_{k}$ to the set $B$. By $(3.2), \zeta_{k}$ are equibounded in $\mathcal{M}_{b}^{+}\left(\Omega^{\prime}\right)$, so, up to a subsequence, $\zeta_{k} \stackrel{*}{\rightarrow} \zeta \in \mathcal{M}_{b}^{+}\left(\Omega^{\prime}\right)$. Recalling Corollary 2.11 and since $\left(\tilde{f}_{p}\right)^{\frac{1}{p}}$ is a norm, we have that

$$
\frac{\mathrm{d}\left(\tilde{f}_{p}\right)^{\frac{1}{p}}(\mathbb{A} \tilde{u})}{\mathrm{d} \mathcal{H}^{n-1}\left\llcorner J_{\tilde{u}}\right.}=\left(\tilde{f}_{p}\right)^{\frac{1}{p}}\left([\tilde{u}] \otimes_{\mathbb{A}} \nu_{\tilde{u}}\right) \quad \mathcal{H}^{n-1} \text {-a.e. in } J_{\tilde{u}} .
$$

Let us fix $x \in J_{\tilde{u}}$ such that the derivatives in 3.15 plus $\frac{\mathrm{d} \zeta}{\mathrm{d} \mathcal{H}^{n-1}\left\llcorner J_{\tilde{u}}\right.}$ exist finite in $x$, and (3.17) is verified in $x$ (this holds for $\mathcal{H}^{n-1}$-a.e. $x \in J_{\tilde{u}}$ ); let

$$
I:=\left\{\varrho \in\left(0, \mathrm{~d}\left(x, \partial \Omega^{\prime}\right)\right): \mu\left(\partial B_{\varrho}(x)\right)=\widehat{\mu}\left(\partial B_{\varrho}(x)\right)=\zeta\left(\partial B_{\varrho}(x)\right)=0\right\} .
$$

For every $\varrho \in\left(0, \mathrm{~d}\left(x, \partial \Omega^{\prime}\right)\right)$ consider the three sets (that partition $\left.B_{\varrho}(x)\right)$

$$
\begin{aligned}
& E_{1}:=B_{\varrho}(x) \cap\left\{\left|\mathbb{A} \tilde{u}_{k}\right| \leq \varrho^{-\frac{1}{2}}\right\} \cap\left\{\tilde{v}_{k} \leq \lambda\right\}, \\
& E_{2}:=B_{\varrho}(x) \cap\left\{\left|\mathbb{A} \tilde{u}_{k}\right|>\varrho^{-\frac{1}{2}}\right\} \cap\left\{\tilde{v}_{k} \leq \lambda\right\}, \\
& E_{3}:=B_{\varrho}(x) \cap\left\{\tilde{v}_{k}>\lambda\right\} .
\end{aligned}
$$

Since, by $\left.\operatorname{HP1} f_{p}\right]$, there exists $C_{f_{p}}^{\prime} \geq C_{f_{p}}>0$ such that $f_{p}(\xi) \leq C_{f_{p}}^{\prime}\left(1+|\xi|^{p}\right)$ and $\tilde{f}_{p}(\xi) \leq C_{f_{p}}^{\prime}|\xi|^{p}$, it holds that

$$
\int_{E_{1}}\left(f_{p}\right)^{\frac{1}{p}}\left(\mathbb{A} \tilde{u}_{k}\right) \mathrm{d} x \leq C_{f_{p}}^{\prime}\left(\omega_{n-1} \varrho^{n}+\varrho^{n-\frac{1}{2}}\right), \quad \int_{E_{1}}\left(\tilde{f}_{p}\right)^{\frac{1}{p}}\left(\mathbb{A} \tilde{u}_{k}\right) \mathrm{d} x \leq C_{f_{p}}^{\prime} \varrho^{n-\frac{1}{2}} .
$$

By $\left(\right.$ HP2 $\left.f_{p}\right)$ We have that

$$
\int_{E_{2}}\left|\left(f_{p}\right)^{\frac{1}{p}}\left(\mathbb{A} \tilde{u}_{k}\right)-\left(\tilde{f}_{p}\right)^{\frac{1}{p}}\left(\mathbb{A} \tilde{u}_{k}\right)\right| \mathrm{d} x \leq \delta_{\varrho} \int_{B_{\varrho}(x) \cap\left\{\tilde{v}_{k} \leq \lambda\right\}}\left|\mathbb{A} \tilde{u}_{k}\right| \mathrm{d} x \leq C\left(C_{f_{p}}\right) \delta_{\varrho} \mu_{k}\left(B_{\varrho}(x)\right),
$$

for

$$
\delta_{\varrho}:=\sup _{s>\varrho^{-1 / 2},|\xi|=1}\left|\frac{\left(f_{p}\right)^{\frac{1}{p}}(s \xi)}{|s|}-\left(\tilde{f}_{p}\right)^{\frac{1}{p}}(\xi)\right|,
$$

using $\left(\overline{\mathrm{HP} 1 f_{p}}\right)$ and the fact that

$$
\sup _{s>\varrho^{-1 / 2}}\left|\frac{\left(f_{p}\right)^{\frac{1}{p}}\left(s \frac{\mathbb{A} \tilde{u}_{k}}{\left|\mathbb{A} \tilde{u}_{k}\right|}\right)}{|s|}-\left(\tilde{f}_{p}\right)^{\frac{1}{p}}\left(\frac{\mathbb{A} \tilde{u}_{k}}{\left|\mathbb{A} \tilde{u}_{k}\right|}\right)\right| \leq \delta_{\varrho} .
$$

By $\left(\right.$ HP2 $\left.f_{p}\right), \lim _{\varrho \rightarrow 0} \delta_{\varrho}=0$ (uniformly in $k$ ). Thus, the estimate $(3.19)$ and the fact that $\lim _{\varrho} \lim _{k} \varrho^{-(n-1)} \mu_{k}\left(B_{\varrho}(x)\right)<C$ (by the choice of $\varrho$ and $x$, in particular $\frac{\mathrm{d} \mu}{\mathrm{d} \mathcal{H}^{n-1}\left\llcorner J_{\tilde{u}}\right.}$ exists finite at $x$ ) give that

$$
\lim _{\substack{\varrho \in I \\ \varrho \rightarrow 0}} \lim _{\substack{k \rightarrow \infty \\ \varrho_{2}}} \varrho_{E_{2}}\left|\left(f_{p}\right)^{\frac{1}{p}}\left(\mathbb{A} \tilde{u}_{k}\right)-\left(\tilde{f}_{p}\right)^{\frac{1}{p}}\left(\mathbb{A} \tilde{u}_{k}\right)\right| \mathrm{d} x=0 .
$$

On the other hand Hölder's inequality gives

$$
\begin{aligned}
\int_{E_{3}}\left(f_{p}\right)^{\frac{1}{p}}\left(\mathbb{A} \tilde{u}_{k}\right) \mathrm{d} x & \leq\left(\int_{E_{3}} f_{p}\left(\mathbb{A} \tilde{u}_{k}\right) \mathrm{d} x\right)^{\frac{1}{p}}\left(\mathcal{L}^{n}\left(E_{3}\right)\right)^{\frac{1}{p^{\prime}}} \\
& \leq \lambda^{-\frac{1}{p}}\left(\zeta_{k}\left(B_{\varrho}(x)\right)\right)^{\frac{1}{p}}\left(\mathcal{L}^{n}\left(B_{\varrho}(x)\right)\right)^{\frac{1}{p^{\prime}}}
\end{aligned}
$$


since

$$
\zeta_{k}\left(B_{\varrho}(x)\right)=D_{k}^{B_{\varrho}(x)}\left(\tilde{u}_{k}, \tilde{v}_{k}\right) \geq \int_{E_{3}}\left(\tilde{v}_{k}+\varepsilon_{k}^{p-1}\right) f_{p}\left(\mathbb{A} \tilde{u}_{k}\right) \mathrm{d} x \geq \lambda \int_{E_{3}} f_{p}\left(\mathbb{A} \tilde{u}_{k}\right) \mathrm{d} x .
$$

Therefore we obtain

$$
\frac{\mathrm{d} \mu}{\mathrm{d} \mathcal{H}^{n-1}\left\llcorner J_{\tilde{u}}\right.}=\lim _{\substack{\varrho \in I \\ \varrho \rightarrow 0}} \lim _{k \rightarrow \infty} \frac{\mu_{k}\left(B_{\varrho}(x)\right)}{\omega_{n-1} \varrho^{n-1}}=\lim _{\substack{\varrho \in I \\ \varrho \rightarrow 0}} \lim _{k \rightarrow \infty} \frac{\widehat{\mu}_{k}\left(B_{\varrho}(x)\right)}{\omega_{n-1} \varrho^{n-1}}=\frac{\mathrm{d} \widehat{\mu}}{\mathrm{d} \mathcal{H}^{n-1}\left\llcorner J_{\tilde{u}}\right.} .
$$

Indeed, the first and the last equalities follow by definition of Radon-Nikodym derivative and the choice of $I$, while the central equality descends by putting together (3.18), (3.21) (divided by $\omega_{n-1} \varrho^{n-1}$ ), and (3.20). In order to deal with (3.21), we remark that $\lim _{\varrho} \lim _{k} \varrho^{-(n-1)} \zeta_{k}\left(B_{\varrho}(x)\right)<C$ since $\frac{\mathrm{d} \zeta}{\mathrm{d} \mathcal{H}^{n-1}\left\llcorner J_{\tilde{u}}\right.}$ exists finite at $x$.

Since $\widehat{\mu}_{k}$ is defined in terms of the convex positively 1-homogeneous $\left(\tilde{f}_{p}\right)^{\frac{1}{p}}$ and $\mathbb{A} \tilde{u}_{k} \stackrel{*}{\rightarrow} \mathbb{A} \tilde{u}$ in $\mathcal{M}_{b}\left(\Omega^{\prime} ; \mathbb{M}_{\text {sym }}^{n \times n}\right)$ by $[3.5)$, Reshetnyak Semicontinuity Theorem (see e.g. [8, Theorem 2.38]) implies that

$$
\left(\tilde{f}_{p}\right)^{\frac{1}{p}}(\mathbb{A} \tilde{u})\left(B_{\varrho}(x)\right) \leq \liminf _{k \rightarrow \infty}\left(\tilde{f}_{p}\right)^{\frac{1}{p}}\left(\mathbb{A} \tilde{u}_{k}\right)\left(B_{\varrho}(x)\right)=\liminf _{k \rightarrow \infty} \widehat{\mu}_{k}\left(B_{\varrho}(x)\right)=\widehat{\mu}\left(B_{\varrho}(x)\right),
$$

if $\varrho \in I$. Taking the Radon-Nikodym derivative of the above inequality with respect to $\mathcal{H}^{n-1}\left\llcorner J_{\tilde{u}}\right.$ at $x$, for $I \ni \varrho \rightarrow 0$, and recalling (3.22) and the choice of $x$ (that gives in particular (3.17) at $x$ ), we deduce (3.15) and then prove the claim 3.14.

We now collect (3.11), 3.12), 3.13), (3.14) and use the arbitrariness of $\lambda \in(0,1)$ (indeed we let $\lambda \rightarrow 0$ ) to conclude the $\Gamma$-lim inf inequality

$$
\tilde{D}(\tilde{u}, \tilde{v}) \leq \liminf _{k \rightarrow \infty} \tilde{D}_{k}\left(\tilde{u}_{k}, \tilde{v}_{k}\right)
$$

that gives the desired inequality $D(u, v) \leq \liminf _{k \rightarrow \infty} D_{k}\left(u_{k}, v_{k}\right)$, by 3.1 .

Moreover, notice that (3.4) gives also the inclusion stated in Theorem 1.1 for the sublevels of $D_{\varepsilon}$. The corresponding compactness property follows arguing as done for proving (3.5), but now the boundedness of the polynomials $\pi_{\Omega^{\prime}} \tilde{u}_{k}$ is a consequence of the fact that $\tilde{u}_{k}=u_{0}$ in $\Omega^{\prime} \backslash \bar{\Omega}$ (we argue separately on each connected component, using Remark 2.8). The convergence of quasi-minimisers for $D_{\varepsilon}$ to a minimiser for $D$ follows by general properties of $\Gamma$-convergence (see e.g. [33, Corollary 7.17]).

Remark 3.1. If $n=2$ and $\mathbb{A}=\mathrm{E}_{D}$, by (3.4) and (2.12) we get still (3.5), as well as (3.10), arguing as done for $n \geq 3$. If we had at disposal the analogous of Theorem 2.9 (and then Corollary 2.11] we could follow the proof of $\Gamma$-lim inf inequality as above.

Remark 3.2. We could reproduce the proof of the $\Gamma$-lim inf inequality above for $n \geq 3$ and the operator

$$
\mathbb{B} u:=\mathrm{E}_{D} u+\frac{\operatorname{div}^{+} u}{n} \mathrm{Id} .
$$

Indeed $B V^{\mathbb{B}}\left(\Omega^{\prime}\right) \subset B V^{\mathrm{E}_{D}}\left(\Omega^{\prime}\right)$, so that $G S B D\left(\Omega^{\prime}\right) \cap B V^{\mathbb{B}}\left(\Omega^{\prime}\right)=S B D\left(\Omega^{\prime}\right)$, and then, applying $\mathbb{B}$ to 2.2 ,

$$
\mathbb{B} u\left\llcorner J_{u}=\left[\left([u] \odot \nu_{u}\right)_{D}+\frac{\left([u] \cdot \nu_{u}\right)^{+}}{n} \operatorname{Id}\right] \mathcal{H}^{n-1}\left\llcorner J_{u}=:[u] \otimes_{\mathbb{B}} \nu_{u} .\right.\right.
$$

Moreover, it holds $\mathbb{B} \tilde{u}_{k}{ }^{*} \mathbb{B} \tilde{u}$ in $\mathcal{M}_{b}\left(\Omega^{\prime} ; \mathbb{M}_{\text {sym }}^{n \times n}\right)$. Thus we get 3.17 for $[u] \otimes_{\mathbb{B}} \nu_{u}$, and so the corresponding version of (3.14). 


\section{4. $\Gamma$-lim sup INEQUALITY}

As in [39], we construct by hand a recovering sequence starting from a function $u$ with regular jump set and smooth outside $J_{u}$. However, since our result is formulated for general $S B D$ functions without requiring a priori integrability for $u$ it is not enough now to apply neither the density results for GSBD [51, Theorem 3.1] and [43, 28, 25], nor the approximations [21, 22] for $S B D$. Indeed all these results do not approximate the jump part of $u$ without assuming $u \in L^{\infty}\left(\Omega ; \mathbb{R}^{n}\right)$ : this request is not natural because the functionals, that depend on $e(u)$, are not decreasing by truncation of $u$.

The analysis is then based on the following approximation for $S B D^{p}$ functions in $B D$ norm, recently proven in [31, Theorem 1.1].

Theorem 4.1. Let $\Omega$ be an open bounded Lipschitz subset of $\mathbb{R}^{n}$, and $u \in S B D^{p}(\Omega)$, with $p>1$. Then there exist $u_{k} \in S B V^{p}\left(\Omega ; \mathbb{R}^{n}\right) \cap L^{\infty}\left(\Omega ; \mathbb{R}^{n}\right)$ such that each $J_{u_{k}}$ is closed and included in a finite union of closed connected pieces of $C^{1}$ hypersurfaces, $u_{k} \in$ $C^{\infty}\left(\bar{\Omega} \backslash J_{u_{k}} ; \mathbb{R}^{n}\right) \cap W^{m, \infty}\left(\Omega \backslash J_{u_{k}} ; \mathbb{R}^{n}\right)$ for every $m \in \mathbb{N}$, and:

$$
\lim _{k \rightarrow \infty}\left(\left\|u_{k}-u\right\|_{B D(\Omega)}+\left\|e\left(u_{k}\right)-e(u)\right\|_{L^{p}\left(\Omega ; \mathbb{M}_{s y m}^{n \times n}\right)}+\mathcal{H}^{n-1}\left(J_{u_{k}} \triangle J_{u}\right)\right)=0 .
$$

We combine the previous approximation with a well-known result by Cortesani and Toader, that allows us to work with the so-called "piecewise smooth" $S B V$-functions, denoted $\mathcal{W}\left(\Omega ; \mathbb{R}^{n}\right)$, namely

$u \in \mathcal{W}\left(\Omega ; \mathbb{R}^{n}\right)$ if $\left\{\begin{array}{l}u \in S B V\left(\Omega ; \mathbb{R}^{n}\right) \cap W^{m, \infty}\left(\Omega \backslash J_{u} ; \mathbb{R}^{n}\right) \text { for every } m \in \mathbb{N}, \\ \mathcal{H}^{n-1}\left(\overline{J_{u}} \backslash J_{u}\right)=0, \\ \overline{J_{u}} \text { is the intersection of } \Omega \text { with a finite union of }(n-1) \text {-dimensional simplexes } .\end{array}\right.$

We report below the result by Cortesani and Toader, in a slightly less general version.

Theorem 4.2 (30], Theorem 3.1). Let $\Omega$ be an open bounded Lipschitz set. For every $u \in S B V^{p}\left(\Omega ; \mathbb{R}^{n}\right) \cap L^{\infty}\left(\Omega ; \mathbb{R}^{n}\right)$ there exist $u_{k} \in \mathcal{W}\left(\Omega ; \mathbb{R}^{n}\right)$ such that

$$
\begin{gathered}
\lim _{k \rightarrow \infty}\left(\left\|u_{k}-u\right\|_{L^{1}\left(\Omega ; \mathbb{R}^{n}\right)}+\left\|\nabla u_{k}-\nabla u\right\|_{L^{p}\left(\Omega ; \mathbb{M}^{n \times n}\right)}+\mathcal{H}^{n-1}\left(J_{u_{k}} \triangle J_{u}\right)\right)=0, \\
\lim _{k \rightarrow \infty} \int_{J_{u_{k}} \cap A} \phi\left(x, u_{k}^{+}, u_{k}^{-}, \nu_{u_{k}}\right) \mathrm{d} \mathcal{H}^{n-1}=\int_{J_{u} \cap A} \phi\left(x, u^{+}, u^{-}, \nu_{u}\right) \mathrm{d} \mathcal{H}^{n-1},
\end{gathered}
$$

for every $A \subset \Omega, \mathcal{H}^{n-1}\left(\partial A \cap J_{u}\right)=0$, and every $\phi$ strictly positive, continuous, and $B V$-elliptic (see e.g. [6] or [30, equation (2.4)] for the notion of BV-ellipticity).

Remark 4.3. In Theorem 4.2 we may assume also $J_{u_{k}} \Subset \Omega$, by [31, Remark 6.3], in turn using [36]. At this stage, [36, Lemma 5.2] gives that for any $p>1$ the $n-1$ dimensional simplexes in the decomposition of $\bar{J}_{u}$ may be taken pairwise disjoint and such that also $J_{u} \cap \Pi_{j} \cap \Pi_{i}=\emptyset$ for any two different hyperplanes $\Pi_{i}, \Pi_{j}$ (if $p \in(1,2]$ it is enough to employ the capacitary argument in [30, Remark 3.5]). Moreover, we notice that our function $\left(\tilde{f}_{p}\right)^{\frac{1}{p}}$ is $B V$-elliptic.

The combination of the density results described so far guarantees that for a given $u \in S B D^{p}(\Omega)$ we can find approximating functions $u_{k} \in \mathcal{W}\left(\Omega, \mathbb{R}^{n}\right)$ with $J_{u_{k}} \Subset \Omega$ and $J_{u_{k}} \cap \Pi_{j} \cap \Pi_{i}=\emptyset$ for any two different hyperplanes $\Pi_{i}, \Pi_{j}$. The last property we have to ensure is that

$$
\operatorname{tr}_{\partial \Omega} u_{k}=\operatorname{tr}_{\partial \Omega} u_{0} \quad \text { on } \partial_{D} \Omega .
$$

This is possible in view of the assumption (1.2), arguing as in [25, Theorem 5.5] with tools from [31], as sketched below. 
For given $u \in S B D^{p}(\Omega)$ and $\varepsilon>0$, one first defines a suitable extension $\widehat{u}_{k}$ of $u$ on $\Omega_{t}:=\Omega+B(0, t)$, for $t<32 k^{-1}$, as follows. We can find pairwise disjoint cubes $\left(Q_{h}\right)_{h=1}^{\bar{h}}$, centered at $x_{h} \in \partial_{N} \Omega$ with sidelength $\varrho_{h}, \mathrm{~d}\left(Q_{h}, \partial_{D} \Omega\right)>d_{\varepsilon}>0$ (recall (1.6)),

$$
\int_{\partial_{N} \Omega \backslash \widehat{Q}} 1+\left|\operatorname{tr} \partial \Omega\left(u-u_{0}\right)\right| \mathrm{d} \mathcal{H}^{n-1}<\varepsilon, \quad D^{\Omega \cap \widehat{Q}}(u, 1)<\eta_{\varepsilon}, \quad \text { for } \widehat{Q}:=\bigcup_{h} \bar{Q}_{h},
$$

for suitable $d_{\varepsilon}, \eta_{\varepsilon}$ with $\lim _{\varepsilon \rightarrow 0} d_{\varepsilon}=\lim _{\varepsilon \rightarrow 0} \eta_{\varepsilon}=0\left(D^{A}\right.$ denotes the energy $D$ in Theorem 1.1 localised on a set $A), J_{u} \cap \partial Q_{h}=\emptyset$ for each $h$,

$$
u \in L^{1}\left(\Omega \cap \bigcup_{h} \partial Q_{h} ; \mathbb{R}^{n}\right), \quad u_{0} \in L^{1}\left(\bigcup_{h} \partial Q_{h} ; \mathbb{R}^{n}\right) .
$$

Moreover, $\partial_{N} \Omega \cap Q_{h}$ is "almost" a diameter of $Q_{h}$ with respect to $\nu_{\partial \Omega}\left(x_{h}\right)$, namely (cf. 31, (4.2)]) there exists $C^{1}$ hypersurfaces $\left(\Gamma_{h}\right)_{h=1}^{\bar{h}}$ with $x_{h} \in \Gamma_{h} \subset Q_{h}$ and

$$
\mathcal{H}^{n-1}\left(\left(\partial_{N} \Omega \triangle \Gamma_{h}\right) \cap \bar{Q}_{h}\right)<\varepsilon\left(2 \varrho_{h}\right)^{n-1}<\frac{\varepsilon}{1-\varepsilon} \mathcal{H}^{n-1}\left(\partial_{N} \Omega \cap \bar{Q}_{h}\right),
$$

$\Gamma_{h}$ is a $C^{1}$ graph with respect to $\nu_{\partial \Omega}\left(x_{h}\right)$ with Lipschitz constant less than $\varepsilon / 2$. Let

$$
\widetilde{u}:=u \chi_{\Omega}+u_{0} \chi_{\Omega_{t} \backslash \Omega},
$$

and notice that by 4.5 we can say that (up to modify $\eta_{\varepsilon}$ )

$$
\int_{\partial_{N} \Omega \backslash \Gamma_{h}}\left|\left[u-u_{0}\right]\right| \mathrm{d} \mathcal{H}^{n-1}=\int_{\partial_{N} \Omega \backslash \Gamma_{h}}|[\widetilde{u}]| \mathrm{d} \mathcal{H}^{n-1}<\eta_{\varepsilon} .
$$

We now approximate $\widetilde{u}$ with respect to the energy $D$, arguing in each $Q_{h}$, by a sequence of functions, depending on a parameter $k$. We notice that the choice of the finite family of cubes $Q_{h}$ is done before the construction of these approximations, and depends only on $\varepsilon$. Then we can argue, as follows, for a fixed cube, denoting $Q \equiv Q_{h}, \Gamma \equiv \Gamma_{h}$ and assuming, up to a rotation and a translation, $x_{h}=0$ and $\nu_{\partial \Omega}\left(x_{h}\right)=e_{n}$ (notice that all the notation indeed depends on $h$ ). Let $Q^{-}$denote the almost half cube contained in $Q$ which is below $\Gamma$ (that is $Q^{-}$is almost contained in $\Omega$ ).

We partition $Q^{-}$in parallelepipeds with first $n-1$ coordinates in squares of sidelength $\left(\eta_{\varepsilon} k\right)^{-1}$

$$
F_{\mathbf{m}}:=\left\{\left(y_{1}, \ldots, y_{n-1}\right) \in \mathbb{R}^{n-1}: y_{i} \in\left(\eta_{\varepsilon} k\right)^{-1} m_{i}+\left(0,\left(\eta_{\varepsilon} k\right)^{-1}\right)\right\}
$$

(we have $\mathbf{m}=\left(m_{1}, \ldots, m_{n-1}\right) \in\left\{-\eta_{\varepsilon} k \varrho,-\eta_{\varepsilon} k \varrho+1, \ldots, 0, \ldots, \eta_{\varepsilon} k \varrho-1\right\}^{n-1} \subset \mathbb{N}^{n-1}$, we may assume $\eta_{\varepsilon} k \varrho \in \mathbb{N}$ ) so that

$$
\Gamma_{h} \cap\left(F_{\mathbf{m}} \times \mathbb{R}\right) \subset F_{\mathbf{m}} \times\left(m_{n}, m_{n}+1 / 2\right) k^{-1},
$$

for some $m_{n} \in \mathbb{N}$ (cf. [31, (4.7), (4.8)]). As in [31, (4.9)], setting $Q_{\mathbf{m}}^{-}:=Q^{-} \cap\left(F_{\mathbf{m}} \times \mathbb{R}\right)$ we use the Nitsche-type extension [31, Lemma 2.1] (see also [54]) to extend $\left.\widetilde{u}\right|_{Q_{\mathrm{m}}^{-}}$along the vertical direction, employing the (part of) hyperplans $F_{\mathbf{m}} \times\left\{m_{n} k^{-1}\right\}$ as the flat interface needed in [31, Lemma 2.1]: we obtain a function $\widetilde{u}_{\mathbf{m}}^{-}$defined on

$$
\widetilde{Q}_{\mathbf{m}}^{-}:=Q \cap\left(F_{\mathbf{m}} \times\left(-\infty,\left(m_{n}+33\right) k^{-1}\right)\right)=F_{\mathbf{m}} \times\left(-\varrho,\left(m_{n}+33\right) k^{-1}\right),
$$

with $\widetilde{u}_{\mathbf{m}}^{-}=\widetilde{u}$ on $\left.F_{\mathbf{m}} \times\left(-\varrho,\left(m_{n}-33\right) k^{-1}\right)\right)$. We do not create a jump on the common boundary between adjacent $Q_{\mathrm{m}}^{-}$except for a region with height of order $k^{-1}$, and this is true also for the jump created with respect to the original $\widetilde{u}$ on the "boundary parallelepipeds", namely the $Q_{\mathbf{m}}^{-}$with $\partial Q_{\mathbf{m}}^{-} \cap \partial Q \neq \emptyset$. With the same arguments of [31, Section 4], one can control both the measure of the union of these small interfaces (by $C \eta_{\varepsilon} \varrho^{n-1}$, see [31, (4.27)]), and the integral of the jump amplitude over this set (cf. [31, (4.32)]). 

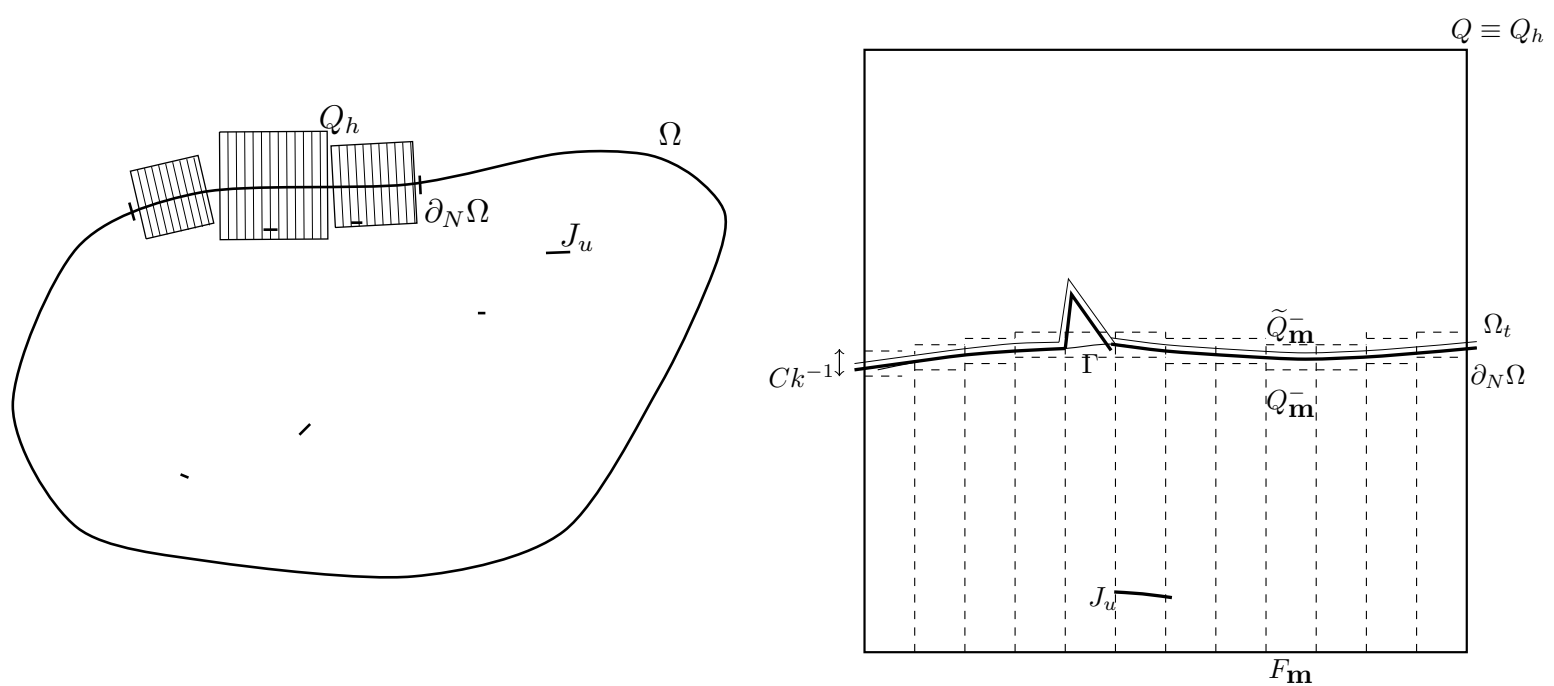

Figure 1 . In the first figure, the cubes $Q_{h}$ covering almost all $\partial_{N} \Omega$. In the second one, a single cube $Q \equiv Q_{h}$ with the relative (almost) parallelepipeds $Q_{\mathbf{m}}^{-}$, their bottom faces $F_{\mathbf{m}}$, and their extensions $\widetilde{Q}_{\mathbf{m}}^{-}$. We see the enlarged domain $\Omega_{t}$, the $C^{1}$ almost diameter $\Gamma$, and the pieces of hyperplanes $F_{\mathbf{m}} \times\left\{m_{n} k^{-1}\right\}$, below $\Gamma$, along which the original function is extended into $\widetilde{u}_{\mathbf{m}}^{-}$. The zones in which we extend $\grave{a}$ la Nitsche have height of order $k^{-1}$.

We obtain that, for a universal $c>1$, (neglect the boundary contribution in $D$ )

$$
D^{\widetilde{Q}_{\mathbf{m}}^{-}}\left(\widetilde{u}_{\mathbf{m}}^{-}, 1\right)<c D^{Q_{\mathbf{m}}^{-}}(\widetilde{u}, 1), \quad D^{\widetilde{Q}_{\mathbf{m}}^{-} \cup \widetilde{Q}_{\mathbf{m}^{\prime}}^{-}}\left(\widetilde{u}_{\mathbf{m}}^{-} \chi_{\widetilde{Q}_{\mathbf{m}}^{-}}^{-}+\widetilde{u}_{\mathbf{m}^{\prime}}^{-} \chi_{\widetilde{Q}_{\mathbf{m}^{\prime}}^{-}}, 1\right)<c D^{Q_{\mathbf{m}}^{-} \cup Q_{\mathbf{m}^{\prime}}^{-}(\widetilde{u}, 1),}
$$

for adjacent $\mathbf{m}, \mathbf{m}^{\prime}$. Notice that, since the extension is done with respect to the vertical direction, for the "boundary parallelepipeds" $Q_{\mathrm{m}}^{-}$we have that

$$
\left\|\operatorname{tr} \widetilde{u}_{\mathbf{m}}^{-}\right\|_{L^{1}(\{\mathrm{~d}(\cdot, \partial \Omega)<t\} \cap \partial Q)} \leq c\|u\|_{L^{1}(\Omega \cap\{\mathrm{d}(\cdot, \partial \Omega)<2 t\} \cap \partial Q)},
$$

which vanishes as $k \rightarrow \infty$, for $\varepsilon>0$, by (4.4) (this is true also taking the union of $\partial Q_{h}$, since $Q_{h}$ are in finite number, independent of $k$ ). Eventually, since $u \in B D$, we are able to estimate the trace of $\widetilde{u}_{\mathbf{m}}^{-}$on $\left.F_{\mathbf{m}} \times\left\{\left(m_{n}+33\right) k^{-1}\right)\right\}$, in terms of the trace of $u$ on $\Gamma$ (cf. e.g. [31, (4.35)]); then we can say that, if $\bar{\Omega}_{t}$ intersects $\left.F_{\mathbf{m}} \times\left\{\left(m_{n}+33\right) k^{-1}\right)\right\}$ (as in the corner for $\partial_{N} \Omega$ in the Figure 1), then

$$
\int_{\left.\left.\left.\left(m_{n}+33\right) k^{-1}\right)\right\}\right)}\left|\operatorname{tr} \widetilde{u}_{\mathbf{m}}^{-}-u_{0}\right| \mathrm{d} \mathcal{H}^{n-1}<\int_{\partial_{N} \Omega \backslash \Gamma_{h}}|[\widetilde{u}]| \mathrm{d} \mathcal{H}^{n-1}+o_{k \rightarrow \infty}(1) .
$$

By 4.3)-4.9) we can see (cf. again [31, (4.16)-(4.34)]) that the extension

$$
\widehat{u}_{k}:= \begin{cases}u & \text { in } \Omega \backslash \widehat{Q}, \\ \widetilde{u}_{\mathbf{m}}^{-} & \text {in } \widetilde{Q}_{\mathbf{m}}^{-}, \text {for any } \mathbf{m} \text { and any } h, \\ u_{0} & \text { elsewhere in } \Omega_{t} .\end{cases}
$$

satisfies (in the following neglect the boundary contribution in $D$ when this is evaluated on $\Omega_{t}, \Omega_{t / 2}$, in other words we treat the boundary outside $\partial_{D} \Omega$ as a Neumann part)

$$
D^{\Omega_{t}}\left(\widehat{u}_{k}, 1\right)<D^{\Omega}(u, 1)+c D^{\Omega \cap \widehat{Q}}(u, 1)+\int_{\partial_{N} \Omega \backslash\left(\cup_{h} \Gamma_{h}\right)} \mid\left[\widetilde{u}|| \mathrm{d} \mathcal{H}^{n-1}+o_{k \rightarrow \infty}(1) .\right.
$$


The vanishing term $o_{k \rightarrow \infty}(1)$ accounts also for the jump created on $\{\mathrm{d}(\cdot, \partial \Omega)<2 t\} \cap$ $\bigcup_{h} \partial Q_{h}$, that is controlled by 4.4 and 4.8), due to the choice of the cubes $Q_{h}$. At this stage, we can follow the strategy of [25, Theorem 5.5]. For $\delta=t / 2=16 k^{-1}$, the function

$$
\widehat{u}_{k}^{\prime}:=\widehat{u}_{k} \circ\left(O_{\delta, x_{0}}\right)^{-1}+u_{0}-u_{0} \circ\left(O_{\delta, x_{0}}\right)^{-1}
$$

is equal to $u_{0}$ in a neighbourhood of $\partial_{D} \Omega$, by (1.2), and satisfies

$$
D^{\Omega_{t / 2}}\left(\widehat{u}_{k}^{\prime}, 1\right)<D^{\Omega_{t}}\left(\widehat{u}_{k}, 1\right)+o_{k \rightarrow \infty}(1) .
$$

Then we apply the construction of [31, Theorem 1.1] to $\widehat{u}_{k}^{\prime}$, to get $\widetilde{u}_{k}$ with

$$
D^{\Omega}\left(\widetilde{u}_{k}, 1\right)<D^{\Omega_{t / 2}}\left(\widehat{u}_{k}^{\prime}, 1\right)+o_{k \rightarrow \infty}(1),
$$

and $\widetilde{u}_{k}=u_{0} * \varrho_{k}$ in a neighbourhood of $\partial_{D} \Omega$, for $\varrho_{k}$ a convolution kernel at scale $k^{-1}$. Eventually, we obtain the approximating function $u_{k}$, satisfying 4.2 and close in energy to $u$, by

$$
u_{k}:=\widetilde{u}_{k}+u_{0}-u_{0} * \varrho_{k} .
$$

We are therefore allowed to start (employing Theorem 4.2 , that preserves the boundary condition, in a neighbourhood of $\left.\partial_{D} \Omega\right)$ from a function $u \in \mathcal{W}\left(\Omega ; \mathbb{R}^{n}\right)$ with $u=u_{0}$ in a neighbourhood of $\partial_{D} \Omega, J_{u} \Subset \Omega$, and it is not restrictive to consider the case $J_{u} \subset \Pi$ for a suitable hyperplane $\Pi$, say $\Pi=\left\{x_{n}=0\right\}$ to fix a simple notation. (From now on we regard $x \in \mathbb{R}^{n}$ as $\left(x^{\prime}, x_{n}\right)$ for $x^{\prime} \in \mathbb{R}^{n-1}$.)

Remark 4.4. To get (4.2) it is enough to assume (1.2) separately for $\partial_{D} \Omega \cap \Omega_{j}$ and suitable $x_{0}^{j} \in \mathbb{R}^{n}, \bar{\delta}^{j}>0$, for each connected component $\Omega_{j}$ of $\Omega$. Moreover, if $p \leq 1^{*}=\frac{n}{n-1}$, the hypothesis (1.2) may be dropped by using partitions of the unity to guarantee the condition (4.2). This is possible since $u \in L^{1^{*}}\left(\Omega ; \mathbb{R}^{n}\right)$, and so $e(\varphi u)=\varphi e(u)+u \odot \nabla \varphi$ is well controlled in $L^{p}$ for any smooth $\varphi$. We also refer to [20] for a corresponding treatment of smooth domains.

Remark 4.5. A variant of Theorem 4.1 with a strong approximation of $\mathbb{A} u$ in $L^{p}$ and $e(u)-\mathbb{A} u$ in $L^{t}$ for functions in $S B D^{p \wedge t}$ would allow us to prove the result for functionals $D_{k}$ depending on $e(u)-\mathbb{A} u$ through a function $g_{t}$ with $t$-growth, $t \neq p$. Unfortunately, following the proof of Theorem 4.1. this would follow from a refined version of [23, Proposition 3] controlling two different powers of $\mathbb{A} u$ and $e(u)-\mathbb{A} u$ : this seems out of reach with the strategy in [23] that relies on slicing properties, useless for $\mathbb{A} u$. For this reason we take $p$ growth both in $\mathbb{A} u$ and in $e(u)-\mathbb{A} u$ (we could consider two different functions $f_{p}$ and $g_{p}$, but it is almost the same, taking $f_{p}$ that acts very differently in the two cases).

Let us now construct a recovery sequence corresponding to a regular $u$, in the sense described above, by adapting the argument in [39, Theorem 3.4]. We set

$$
\sigma_{k}(x):=\frac{\varepsilon_{k}}{2 p^{\prime} \psi^{\frac{1}{p^{\prime}}}(0)} p^{\frac{1}{p}}\left(p^{\prime}\right)^{\frac{1}{p^{\prime}}}\left(\tilde{f}_{p}\right)^{\frac{1}{p}}\left(\left|[u]\left(x^{\prime}, 0\right) \otimes_{\mathbb{A}} e_{n}\right|\right) \quad \text { for } x \in J_{u}=J_{u} \cap \Pi .
$$

Since $u$ is Lipschitz up to $J_{u}$, then also $\sigma_{k}$ is Lipschitz with

$$
\left|\nabla \sigma_{k}\right| \leq C \varepsilon_{k},
$$

where $C>0$ depends on the Lipschitz constant of $u, f_{p}, \mathbb{A}$. As in [39], let for any $\varrho<1$

$$
h_{1}(\varrho):=\psi(1-\varrho), \quad h_{2}(\varrho):=\left(\int_{0}^{1-\varrho} \psi^{-\frac{1}{q}}(s) \mathrm{d} s\right)^{-1}, \quad h(\varrho):=h_{1} h_{2}(\varrho) .
$$

Since $\psi$ is positive and vanishing in 1 , we have that $h$ is increasing and vanishing in 0 and $\frac{h_{1}}{h_{2}}$ also vanishes in 0 , so that $\varrho_{k}:=h^{-1}\left(\varepsilon_{k}\right)$ is vanishing and

$$
\lim _{k \rightarrow \infty} \frac{h_{1}\left(\varrho_{k}\right)}{\varepsilon_{k}}=\lim _{k \rightarrow \infty} \frac{\varepsilon_{k}}{h_{2}\left(\varrho_{k}\right)}=0 .
$$


Let $w_{k}$ be the unique solution to the Cauchy problem

$$
\left\{\begin{array}{l}
w_{k}^{\prime}=\left(\frac{q^{\prime}}{\gamma q}\right)^{\frac{1}{q}} \varepsilon_{k}^{-1} \psi^{\frac{1}{q}}\left(w_{k}\right) \\
w_{k}(0)=0
\end{array}\right.
$$

in $\left[0, T_{k}\right)$, where $T_{k}:=\left(\frac{\gamma q}{q^{\prime}}\right)^{\frac{1}{q}} \varepsilon_{k} \int_{0}^{1} \psi^{-1 / q}(s) \mathrm{d} s \in(0, \infty]$. We have that $w_{k}$ is the inverse of the function

$$
z \in\left(\varepsilon_{k}, 1\right] \mapsto\left(\frac{\gamma q}{q^{\prime}}\right)^{\frac{1}{q}} \varepsilon_{k} \int_{0}^{z} \psi^{-1 / q}(s) \mathrm{d} s,
$$

in $\left[0, T_{k}\right)$. Let $\tau_{k}:=w_{k}^{-1}\left(1-\varrho_{k}\right)$, namely

$$
\tau_{k}=\left(\frac{\gamma q}{q^{\prime}}\right)^{\frac{1}{q}} \varepsilon_{k} \int_{0}^{1-\varrho_{k}} \psi^{-1 / q}(s) \mathrm{d} s \in\left(0, T_{k}\right),
$$

which is infinitesimal in view of 4.12, and define the sets

$$
\begin{aligned}
& A_{k}:=\left\{x \in \mathbb{R}^{n}:\left(x^{\prime}, 0\right) \in J_{u},\left|x_{n}\right|<\sigma_{k}\left(x^{\prime}\right)\right\}, \\
& B_{k}:=\left\{x \in \mathbb{R}^{n}:\left(x^{\prime}, 0\right) \in J_{u}, 0 \leq\left|x_{n}\right|-\sigma_{k}\left(x^{\prime}\right) \leq \tau_{k}\right\}, \\
& C_{k}:=\left\{x \in \mathbb{R}^{n}:\left(x^{\prime}, 0\right) \notin J_{u}, \mathrm{~d}\left(x, J_{u}\right) \leq \tau_{k}\right\} .
\end{aligned}
$$

The candidate recovery sequence $\left(u_{k}, v_{k}\right)$ is then

$$
u_{k}(x):= \begin{cases}\frac{x_{n}+\sigma_{k}\left(x^{\prime}\right)}{2 \sigma_{k}\left(x^{\prime}\right)}\left(u\left(x^{\prime}, \sigma_{k}\left(x^{\prime}\right)\right)-u\left(x^{\prime},-\sigma_{k}\left(x^{\prime}\right)\right)\right)+u\left(x^{\prime},-\sigma_{k}\left(x^{\prime}\right)\right), & \text { if } x \in A_{k}, \\ u(x) & \text { if } x \notin A_{k}\end{cases}
$$

and (recall that in the functional there are $v+\varepsilon_{k}$ and $v+\eta_{\varepsilon_{k}}$ )

$$
v_{k}(x):= \begin{cases}0 & \text { if } x \in A_{k}, \\ w_{k}\left(\left|x_{n}\right|-\sigma_{k}\left(x^{\prime}\right)\right) & \text { if } x \in B_{k}, \\ w_{k}\left(\mathrm{~d}\left(x, J_{u}\right)\right) & \text { if } x \in C_{k}, \\ 1-\varrho_{k} & \text { otherwise. }\end{cases}
$$

It is immediate that the sequences $\left(u_{k}\right)_{k}$ and $\left(v_{k}\right)_{k}$ converge pointwise to $u$ and 1 . Moreover, for the components $u_{k}^{i}$ of $u_{k}$,

$$
\partial_{n} u_{k}^{i}(x)=\frac{u^{i}\left(x^{\prime}, \sigma_{k}\left(x^{\prime}\right)\right)-u^{i}\left(x^{\prime},-\sigma_{k}\left(x^{\prime}\right)\right)}{2 \sigma_{k}\left(x^{\prime}\right)}, \quad i=1, \ldots, n,
$$

and, by straightforward calculations (see also [39])

$$
\begin{gathered}
\left|\partial_{j} u_{k}^{i}(x)\right| \leq\left|\partial_{j} \sigma_{k}\left(x^{\prime}\right)\right|\left(\frac{\left|\left[u^{i}\right]\left(x^{\prime}, 0\right)\right|}{2 \sigma_{k}\left(x^{\prime}\right)}+4 L\right)+3 L \leq C \text { for } j=1, \ldots, n-1, \\
\left|\partial_{n} u_{k}^{i}(x)\right| \leq L+\frac{\left|\left[u^{i}\right]\left(x^{\prime}, 0\right)\right|}{2 \sigma_{k}\left(x^{\prime}\right)} \leq \frac{C}{\varepsilon_{k}},
\end{gathered}
$$

in $A_{k}$, where $L$ is the Lipschitz constant of $u$ in $\Omega \backslash J_{u}$ and $C$ depends on $L$ (recall also (4.11)). By the way, $u_{k}$ is a Lipschitz function. Notice also that

$$
\lim _{k \rightarrow \infty}\left|\partial_{n} u_{k}^{i}(x)\right|=\infty \quad \text { for } x \in J_{u} .
$$

Let us estimate the energy $D_{k}\left(u_{k}, v_{k}\right)$. We have that

$$
\limsup _{k \rightarrow \infty} \int_{\Omega \backslash A_{k}}\left(v_{k}+\varepsilon_{k}^{p-1}\right) f_{p}\left(\mathbb{A} u_{k}\right) \mathrm{d} x=\limsup _{k \rightarrow \infty} \int_{\Omega \backslash A_{k}}\left(v_{k}+\varepsilon_{k}^{p-1}\right) f_{p}(\mathbb{A} u) \mathrm{d} x \leq \int_{\Omega} f_{p}(\mathbb{A} u) \mathrm{d} x,
$$


and

$$
\limsup _{k \rightarrow \infty} \int_{\Omega \backslash A_{k}}\left(v_{k}+\eta_{\varepsilon_{k}}\right) f_{p}\left(e\left(u_{k}\right)-\mathbb{A} u_{k}\right) \mathrm{d} x \leq \int_{\Omega} f_{p}(e(u)-\mathbb{A} u) \mathrm{d} x .
$$

Now, recalling (4.14), we get

$\int_{A_{k}}\left(v_{k}+\eta_{\varepsilon_{k}}\right) f_{p}\left(e\left(u_{k}\right)-\mathbb{A} u_{k}\right) \mathrm{d} x=\int_{A_{k}} \eta_{\varepsilon_{k}} f_{p}\left(e\left(u_{k}\right)-\mathbb{A} u_{k}\right) \mathrm{d} x \leq \mathcal{L}^{n}\left(A_{k}\right) \eta_{\varepsilon_{k}} \frac{C^{p}}{\left(\varepsilon_{k}\right)^{p}} \leq C \frac{\eta_{\varepsilon_{k}}}{\left(\varepsilon_{k}\right)^{p-1}}$,

and this tends to 0 since $\lim _{\varepsilon \rightarrow 0} \frac{\eta_{\varepsilon}}{\varepsilon^{p-1}}=0$.

In view of the fact that $\lim _{k \rightarrow \infty} \mathcal{L}^{n}\left(A_{k}\right)=0$ and of the estimates for the tangential derivatives 4.14), we do not see the contribution of the tangential derivatives in the limit. Moreover, 4.15) and assumption $\left(\overline{\left.\operatorname{HP} 2 f_{p}\right)}\right.$ allows us to replace $f_{p}$ with $\tilde{f}_{p}$ for the normal derivatives, in the limit. Then (see (1.5))

$$
\limsup _{k \rightarrow \infty} \int_{A_{k}}\left(v_{k}+\varepsilon_{k}^{p-1}\right) f_{p}\left(\mathbb{A} u_{k}\right) \mathrm{d} x=\limsup _{k \rightarrow \infty} \int_{A_{k}} \varepsilon_{k}^{p-1} \tilde{f}_{p}\left(\partial_{n} u_{k} \otimes_{\mathbb{A}} e_{n}\right) \mathrm{d} x
$$

for $\partial_{n} u_{k}$ the vector of the normal derivatives in (4.13) and $e_{n}=(0, \ldots, 0,1) \in \mathbb{R}^{n}$. By 4.13 and the fact that $\tilde{f}_{p}$ is positively $p$-homogeneous we get

$$
\begin{aligned}
& \limsup _{k \rightarrow \infty} \int_{A_{k}}\left(v_{k}+\varepsilon_{k}^{p-1}\right) f_{p}\left(\mathbb{A} u_{k}\right) \mathrm{d} x \\
& \quad=\limsup _{k \rightarrow \infty} \int_{A_{k}} \frac{\varepsilon_{k}^{p-1}}{\left(2 \sigma_{k}\left(x^{\prime}\right)\right)^{p}} \tilde{f}_{p}\left(\left(u\left(x^{\prime}, \sigma_{k}\left(x^{\prime}\right)\right)-u\left(x^{\prime},-\sigma_{k}\left(x^{\prime}\right)\right)\right) \otimes_{\mathbb{A}} e_{n}\right) \mathrm{d} \mathcal{H}^{n-1}\left(x^{\prime}\right) .
\end{aligned}
$$

Recalling the definitions of $\sigma_{k}$ and $A_{k}$, the pointwise convergence of $u\left(x^{\prime}, \sigma_{k}\left(x^{\prime}\right)\right)-u\left(x^{\prime},-\sigma_{k}\left(x^{\prime}\right)\right)$ to $[u](x) \equiv[u]\left(x^{\prime}\right)$, a change of variables and the Dominated Convergence Theorem give that the terms above are equal to

$$
\int_{J_{u}} \lim _{k \rightarrow \infty} \frac{\varepsilon_{k}^{p-1}}{\left(2 \sigma_{k}\left(x^{\prime}\right)\right)^{p-1}} \tilde{f}_{p}\left([u]\left(x^{\prime}\right) \otimes_{\mathbb{A}} e_{n}\right) \mathrm{d} \mathcal{H}^{n-1}\left(x^{\prime}\right)
$$

and then to

$$
\frac{p^{1 / p}\left(p^{\prime}\right)^{1 / p^{\prime}} \psi(0)^{1 / p}}{p} \int_{J_{u}}\left(\tilde{f}_{p}\right)^{\frac{1}{p}}\left([u] \otimes_{\mathbb{A}} e_{n}\right) \mathrm{d} \mathcal{H}^{n-1}
$$

since

$$
\left(p^{\prime}\right)^{\left(1-1 / p^{\prime}\right)(p-1)}=\left(p^{\prime}\right)^{1 / p^{\prime}}, \quad p^{-\frac{p-1}{p}}=p^{1 / p} / p .
$$

As for the remaining terms of $D_{k}$, notice that by the definition of $v_{k}$ and 4.12 we have

$$
\begin{aligned}
\limsup _{k \rightarrow \infty} \int_{\Omega}\left[\frac{\psi\left(v_{k}\right)}{\varepsilon_{k}}+\gamma \varepsilon_{k}^{q-1}\left|\nabla v_{k}\right|^{q}\right] \mathrm{d} x \leq & \limsup _{k \rightarrow \infty} \int_{B_{k} \cup C_{k}}\left[\frac{\psi\left(v_{k}\right)}{\varepsilon_{k}}+\gamma \varepsilon_{k}^{q-1}\left|\nabla v_{k}\right|^{q}\right] \mathrm{d} x \\
& +\limsup _{k \rightarrow \infty} \int_{A_{k}} \frac{\psi\left(v_{k}\right)}{\varepsilon_{k}} \mathrm{~d} x .
\end{aligned}
$$


We deduce now that

$$
\begin{aligned}
\limsup _{k \rightarrow \infty} \int_{B_{k}} & {\left[\frac{\psi\left(v_{k}\right)}{\varepsilon_{k}}+\gamma \varepsilon_{k}^{q-1}\left|\nabla v_{k}\right|^{q}\right] \mathrm{d} x=\limsup _{k \rightarrow \infty} \int_{J_{u}}\left(\int_{0}^{\tau_{k}}\left[\frac{\psi\left(w_{k}\right)}{\varepsilon_{k}}+\gamma \varepsilon_{k}^{q-1}\left(w_{k}^{\prime}\right)^{q}\right] \mathrm{d} x_{n}\right) \mathrm{d} \mathcal{H}^{n-1}\left(x^{\prime}\right) } \\
= & 2\left(q^{\prime}\right)^{1 / q^{\prime}}(\gamma q)^{1 / q} \limsup _{k \rightarrow \infty} \int_{J_{u}}\left(\int_{0}^{\tau_{k}} \psi^{1 / q^{\prime}}\left(w_{k}\right) w_{k}^{\prime} \mathrm{d} x_{n}\right) \mathrm{d} \mathcal{H}^{n-1}\left(x^{\prime}\right) \\
& =2\left(q^{\prime}\right)^{1 / q^{\prime}}(\gamma q)^{1 / q} \limsup _{k \rightarrow \infty}\left(\int_{0}^{1-\varrho_{k}} \psi^{1 / q^{\prime}}(s) \mathrm{d} s\right) \mathcal{H}^{n-1}\left(J_{u}\right) \\
& =a \mathcal{H}^{n-1}\left(J_{u}\right) .
\end{aligned}
$$

Indeed in the first equality we have used the estimate (4.11) to neglect the contribution of the tangential derivatives of $v_{k}$ in the limit, and the second one follows from the definition of $w_{k}\left(w_{k}^{\prime}\right.$ represents the normal derivative of $\left.v_{k}\right)$ that gives $\alpha^{q}=\beta^{q^{\prime}}$, that is the condition to have the Young equality $\frac{\alpha^{q}}{q}+\frac{\beta^{q^{\prime}}}{q^{\prime}}=\alpha \beta$, for (recall (3.6) $)$

$$
\alpha=\left(\gamma q \varepsilon_{k}^{q-1}\left(w_{k}^{\prime}\right)^{q}\right)^{1 / q}, \quad \beta=\left(q^{\prime} \psi\left(w_{k}\right) \varepsilon_{k}^{-1}\right)^{1 / q^{\prime}} .
$$

Furthermore, arguing similarly and using the Coarea formula (cf. [39, eq. (4.49)]) we get

$$
\int_{C_{k}}\left[\frac{\psi\left(v_{k}\right)}{\varepsilon_{k}}+\gamma \varepsilon_{k}^{q-1}\left|\nabla v_{k}\right|^{q}\right] \mathrm{d} x \leq C \mu_{k} \int_{0}^{1-\varrho_{k}} \psi^{1 / q^{\prime}}(s) \mathrm{d} s \leq C \mu_{k},
$$

so that

$$
\limsup _{k \rightarrow \infty} \int_{C_{k}}\left[\frac{\psi\left(v_{k}\right)}{\varepsilon_{k}}+\gamma \varepsilon_{k}^{q-1}\left|\nabla v_{k}\right|^{q}\right] \mathrm{d} x=0
$$

Eventually

$$
\int_{A_{k}} \frac{\psi\left(v_{k}\right)}{\varepsilon_{k}} \mathrm{~d} x=\int_{J_{u}} \frac{2 \sigma_{k}\left(x^{\prime}\right)}{\varepsilon_{k}} \psi(0) \mathrm{d} \mathcal{H}^{n-1}\left(x^{\prime}\right)=\frac{p^{1 / p}\left(p^{\prime}\right)^{1 / p^{\prime}} \psi(0)^{1 / p}}{p^{\prime}} \int_{J_{u}}\left(\tilde{f}_{p}\right)^{\frac{1}{p}}\left([u] \otimes_{\mathbb{A}} e_{n}\right) \mathrm{d} \mathcal{H}^{n-1} .
$$

Collecting all the estimates below 4.15 we then conclude the $\Gamma$-lim sup inequality.

Remark 4.6. With the notation of Remark 3.2, we could reproduce also the proof of the $\Gamma$-limsup inequality for $\mathbb{B}$ in place of $\mathbb{A}$. Indeed, we define $\sigma_{k}$ and $u_{k}$ in terms of $\mathbb{B}$, and notice that in 4.16) we see in the limit $\left(\partial_{n} u_{k} \odot e_{n}\right)_{D}$ plus the contribution of $\left(\partial_{n} u_{k}^{n}\right)^{+}$, asymptotically equal to that of $\operatorname{div}^{+} u_{k}$ by (4.14). Now $2 \sigma_{k}\left(\partial_{n} u_{k} \odot e_{n}\right)_{D}$ converge pointwise to $\left([u] \odot e_{n}\right)_{D}$ and $2 \sigma_{k}\left(\partial_{n} u_{k}^{n}\right)^{+}$to $\left[u^{n}\right]^{+}=\left([u] \cdot e_{n}\right)^{+}$, which gives $\mathbb{B} u$ in the limit, according to $(3.23)$.

Acknowledgements. Vito Crismale has been supported by the Marie Skłodowska-Curie Standard European Fellowship No. 793018, and by a public grant as part of the Investissement d'avenir project, reference ANR-11-LABX-0056-LMH, LabEx LMH.

\section{REFERENCES}

[1] R. Alessi, M. Ambati, T. Gerasimov, S. Vidoli, and L. De Lorenzis, Comparison of PhaseField Models of Fracture Coupled with Plasticity, Springer International Publishing, Cham, 2018, pp. $1-21$.

[2] R. Alessi, J.-J. Marigo, C. Maurini, and S. Vidoli, Coupling damage and plasticity for a phasefield regularisation of brittle, cohesive and ductile fracture: one-dimensional examples, Int. J. Mech. Sci., 149 (2018), pp. 559-576.

[3] R. Alicandro, A. Braides, And J. Shah, Free-discontinuity problems via functionals involving the $L^{1}$-norm of the gradient and their approximations, Interfaces Free Bound., 1 (1999), pp. 17-37.

[4] S. Almi, Energy release rate and quasi-static evolution viavanishing viscosity in a fracture model depending on the crack opening, ESAIM Control Optim. Calc. Var., 23 (2017), pp. 791-826. 
[5] M. Ambati, T. Gerasimov, and L. De Lorenzis, A review on phase-field models of brittle fracture and a new fast hybrid formulation, Comput. Mech., 55 (2015), pp. 383-405.

[6] L. Ambrosio, Existence theory for a new class of variational problems, Arch. Rational Mech. Anal., 111 (1990), pp. 291-322.

[7] L. Ambrosio, A. Coscia, And G. Dal Maso, Fine properties of functions with bounded deformation, Arch. Ration. Mech. Anal., 139 (1997), pp. 201-238.

[8] L. Ambrosio, N. Fusco, And D. Pallara, Functions of bounded variation and free discontinuity problems, Oxford Mathematical Monographs, The Clarendon Press, Oxford University Press, New York, 2000.

[9] L. Ambrosio and V. M. Tortorelli, Approximation of functionals depending on jumps by elliptic functionals via $\Gamma$-convergence, Comm. Pure Appl. Math., 43 (1990), pp. 999-1036.

[10] A. Arroyo-Rabasa, G. D. Philippis, J. Hirsch, And F. Rindler, Dimensional estimates and rectifiability for measures satisfying linear pde constraints, 2018.

[11] J.-F. Babadjian, Traces of functions of bounded deformation, Indiana Univ. Math. J., 64 (2015), pp. 1271-1290.

[12] M. Barchiesi, G. Lazzaroni, and C. I. Zeppieri, A bridging mechanism in the homogenization of brittle composites with soft inclusions, SIAM J. Math. Anal., 48 (2016), pp. 1178-1209.

[13] G. I. Barenblatt, The mathematical theory of equilibrium cracks in brittle fracture, in Advances in Applied Mechanics, Vol. 7, Academic Press, New York, 1962, pp. 55-129.

[14] G. Bellettini, A. Coscia, And G. Dal Maso, Compactness and lower semicontinuity properties in $\operatorname{SBD}(\Omega)$, Math. Z., 228 (1998), pp. 337-351.

[15] B. Bourdin, Numerical implementation of the variational formulation for quasi-static brittle fracture, Interfaces Free Bound., 9 (2007), pp. 411-430.

[16] B. Bourdin, G. A. Francfort, And J.-J. Marigo, Numerical experiments in revisited brittle fracture, J. Mech. Phys. Solids, 48 (2000), pp. 797-826.

[17] D. Breit, L. Diening, And F. Gmeineder, Traces of functions of bounded $\mathbb{A}$-variation and variational problems with linear growth, Preprint arXiv:1707.06804 (2017).

[18] S. Burke, C. Ortner, And E. SÜLI, An adaptive finite element approximation of a generalized Ambrosio-Tortorelli functional, Math. Models Methods Appl. Sci., 23 (2013), pp. 1663-1697.

[19] G. Buttazzo, Semicontinuity, relaxation and integral representation in the calculus of variations, vol. 207 of Pitman Research Notes in Mathematics Series, Longman Scientific \& Technical, Harlow; copublished in the United States with John Wiley \& Sons, Inc., New York, 1989.

[20] M. Caroccia And N. VAn Goethem, Damage-driven fracture with low-order potentials: asymptotic behavior and applications, 2018, Preprint arXiv:1712.08556

[21] A. Chambolle, An approximation result for special functions with bounded deformation, J. Math. Pures Appl. (9), 83 (2004), pp. 929-954.

[22] — - Addendum to: "An approximation result for special functions with bounded deformation" [J. Math. Pures Appl. (9) 83 (2004), no. 7, 929-954; mr2074682], J. Math. Pures Appl. (9), 84 (2005), pp. $137-145$.

[23] A. Chambolle, S. Conti, And G. A. Francfort, Korn-Poincaré inequalities for functions with a small jump set, Indiana Univ. Math. J., 65 (2016), pp. 1373-1399.

$[24]$ - Approximation of a brittle fracture energy with a constraint of non-interpenetration, Arch. Ration. Mech. Anal., 228 (2018), pp. 867-889.

[25] A. Chambolle and V. CRismale, A density result in GSBD ${ }^{p}$ with applications to the approximation of brittle fracture energies, In press on Arch. Ration. Mech. Anal. (2018), https://doi.org/10.1007/s00205-018-01344-7.

[26] — Compactness and lower semicontinuity in GSBD, Preprint arXiv:1802.03302 (2018), accepted for publication on J. Eur. Math. Soc. (JEMS).

[27] S. Conti, M. Focardi, And F. Iurlano, Phase field approximation of cohesive fracture models, Ann. Inst. H. Poincaré Anal. Non Linéaire, 33 (2016), pp. 1033-1067.

[28] _ Approximation of fracture energies with p-growth via piecewise affine finite elements, Accepted for publication on ESAIM Control Optim. Calc. Var. DOI 10.1051/cocv/2018021.

[29] — Existence of strong minimizers for the Griffith static fracture model in dimension two, In press on Ann. Inst. H. Poincaré Anal. Non Linéaire (2018).

[30] G. Cortesani and R. Toader, A density result in SBV with respect to non-isotropic energies, Nonlinear Anal., 38 (1999), pp. 585-604.

[31] V. Crismale, On the approximation of $S B D$ functions and some applications, Preprint arXiv:1806.03076 (2018).

[32] S. DAIn, Generalized Korn's inequality and conformal Killing vectors, Calc. Var. Partial Differential Equations, 25 (2006), pp. 535-540.

[33] G. Dal Maso, An introduction to $\Gamma$-convergence, vol. 8 of Progress in Nonlinear Differential Equations and their Applications, Birkhäuser Boston, Inc., Boston, MA, 1993. 
[34] — Generalised functions of bounded deformation, J. Eur. Math. Soc. (JEMS), 15 (2013), pp. 19431997.

[35] G. Dal Maso, G. Orlando, and R. Toader, Fracture models for elasto-plastic materials as limits of gradient damage models coupled with plasticity: the antiplane case, Calc. Var. Partial Differential Equations, 55 (2016), pp. Art. 45, 39.

[36] G. De Philippis, N. Fusco, And A. Pratelli, On the approximation of SBV functions, Atti Accad. Naz. Lincei Rend. Lincei Mat. Appl., 28 (2017), pp. 369-413.

[37] G. De Philippis and F. Rindler, On the structure of $\mathcal{A}$-free measures and applications, Ann. of Math. (2), 184 (2016), pp. 1017-1039.

[38] H. Federer, Geometric measure theory, Die Grundlehren der mathematischen Wissenschaften, Band 153, Springer-Verlag New York Inc., New York, 1969.

[39] M. Focardi And F. Iurlano, Asymptotic analysis of Ambrosio-Tortorelli energies in linearized elasticity, SIAM J. Math. Anal., 46 (2014), pp. 2936-2955.

[40] I. Fonseca And N. Fusco, Regularity results for anisotropic image segmentation models, Ann. Scuola Norm. Sup. Pisa Cl. Sci. (4), 24 (1997), pp. 463-499.

[41] I. Fonseca And S. Müller, Quasi-convex integrands and lower semicontinuity in $L^{1}$, SIAM J. Math. Anal., 23 (1992), pp. 1081-1098.

[42] G. A. Francfort and J.-J. Marigo, Revisiting brittle fracture as an energy minimization problem, J. Mech. Phys. Solids, 46 (1998), pp. 1319-1342.

[43] M. Friedrich, A Piecewise Korn Inequality in SBD and Applications to Embedding and Density Results, SIAM J. Math. Anal., 50 (2018), pp. 3842-3918.

[44] M. Fuchs And S. Repin, Some Poincaré-type inequalities for functions of bounded deformation involving the deviatoric part of the symmetric gradient, Zap. Nauchn. Sem. S.-Peterburg. Otdel. Mat. Inst. Steklov. (POMI), 385 (2010), pp. 224-233, 237.

[45] A. Giacomini, Ambrosio-Tortorelli approximation of quasi-static evolution of brittle fractures, Calc. Var. Partial Differential Equations, 22 (2005), pp. 129-172.

[46] F. Gmeineder and B. Raita, Embeddings for $\mathbb{A}$-weakly differentiable functions on domains, Preprint arXiv:1709.04508 (2017).

[47] - On critical $L^{p}$-differentiability of BD-maps, Preprint arXiv:1802.10364 (2018), To appear on Rev. Mat. Iberoam.

[48] A. A. Griffith, The phenomena of rupture and flow in solids, Philos. Trans. Roy. Soc. London Ser. A, 221 (1920), pp. 163-198.

[49] J. W. Hutchinson, A course on nonlinear fracture mechanics, Department of Solid Mechanics, Techn. University of Denmark, 1989.

[50] F. Iurlano, Fracture and plastic models as $\Gamma$-limits of damage models under different regimes, Adv. Calc. Var., 6 (2013), pp. 165-189.

[51] — A density result for GSBD and its application to the approximation of brittle fracture energies, Calc. Var. Partial Differential Equations, 51 (2014), pp. 315-342.

[52] D. Mumford and J. Shah, Boundary detection by minimizing functionals. Proc. IEEE Conf. on Computer Vision and Pattern Recognition, San Francisco, 1985.

[53] M. NegRI, A non-local approximation of free discontinuity problems in $S B V$ and $S B D$, Calc. Var. Partial Differential Equations, 25 (2006), pp. 33-62.

[54] J. A. Nitsche, On Korn's second inequality, RAIRO Anal. Numér., 15 (1981), pp. 237-248.

[55] W. F. OsGood, Note on the functions defined by infinite series whose terms are analytic functions of a complex variable; with corresponding theorems for definite integrals, Ann. of Math. (2), 3 (1901/02), pp. 25-34.

[56] L. Simon, Lectures on geometric measure theory, vol. 3 of Proceedings of the Centre for Mathematical Analysis, Australian National University, Australian National University, Centre for Mathematical Analysis, Canberra, 1983.

[57] D. Spector and J. V. Schaftingen, Optimal embeddings into Lorentz spaces for some vector differential operators via Gagliardo's lemma, 2018.

[58] R. Temam, Mathematical problems in plasticity, Gauthier-Villars, Paris, 1985. Translation of Problèmes mathématiques en plasticité. Gauthier-Villars, Paris, 1983.

[59] J. VAn Schaftingen, Limiting Sobolev inequalities for vector fields and canceling linear differential operators, J. Eur. Math. Soc. (JEMS), 15 (2013), pp. 877-921. 